\title{
Electromagnetic field and short-range wake function in a beam pipe of elliptical cross section
}

\author{
A. Lutman* and R. Vescovo ${ }^{\dagger}$ \\ Università degli Studi di Trieste, DEEI, Trieste, Italy \\ P. Craievich \\ Sincrotrone Trieste, ELETTRA, Trieste, Italy \\ (Received 18 April 2008; published 14 July 2008)
}

\begin{abstract}
Within the ultrarelativistic limit, analytical expressions are found for the high-frequency resistive-wall coupling impedance of an elliptical cross-section vacuum chamber. Subsequently, the corresponding wake functions are derived by performing inverse Fourier transformations numerically. The electromagnetic fields have been developed working out two systems of solutions, namely for the vacuum and for the resistive wall. The constants involved in these systems have been determined by matching boundary conditions at the interface vacuum wall. Several study cases have been considered concerning the aspect ratio of the elliptical cross section and the transverse position of the leading charge in order to exemplify the behavior of the longitudinal and transverse wake functions.
\end{abstract}

DOI: 10.1103/PhysRevSTAB.11.074401

PACS numbers: 41.60.- m, 03.50.De

\section{INTRODUCTION}

Free electron laser (FEL) projects aim to achieve highbrightness photon beam pulses of minimum bandwidth. However, such pulses may be corrupted by possible large wakefields along the undulator small-gap vacuum chamber. Thus, knowledge of the short-range wakefields in the undulator vacuum chamber is needed to predict the beam quality in terms of the single bunch energy spread and emittance. A possible choice for a small-gap vacuum chamber is one with elliptical cross section, for which there are references to analytically derived expressions for the low-frequency resistive-wall coupling impedance (see for example [1-3]). Expressions for the highfrequency resistive-wall impedance are given, in the case of DC conductivity model, for a round pipe in [4], and for the conducting parallel plates in [5]. Adopting the AC conductivity model [6], the resistive-wall impedance is given for the round pipe in [7] and for the parallel plates in [8]. The problem of calculating the coupling impedance, including the high frequencies, of a resistive beam pipe with arbitrary cross section has been solved by Yokoya [9] with the boundary element method. He applied the method to numerically work out the solution in the case of an elliptical pipe; moreover, the method can be applied using both AC and DC conductivity models.

In this paper we analytically derive expressions for the longitudinal and transverse resistive-wall coupling impedance of a vacuum chamber with elliptical cross section

\footnotetext{
*alberto.lutman@elettra.trieste.it

†vescovo@univ.trieste.it

paolo.craievich@elettra.trieste.it
}

using another method, precisely the field matching method, and make a comparison with the results obtained with the boundary element method on the same geometry. The paper is organized as follows. In Sec. II we describe the physical model used to obtain the expressions of the electromagnetic fields inside the vacuum and inside the resistive parts of the beam pipe, respectively. We use elliptical coordinates and write the field components in term of Fourier transformations. In Sec. III we work out the Maxwell equations to derive a series expression of the electromagnetic (e.m.) field in the vacuum part, while in Sec. IV we derive a series expansion of the e.m. field in the resistive part of the beam pipe. In Sec. V, the constants involved in the two series expansions are determined by imposing the boundary conditions at the interface vacuumresistive wall. This fully determines the field components. In Sec. VI we derive the expressions of the longitudinal and transverse resistive-wall impedances, and the short-range wake functions are then obtained by calculating numerically the inverse Fourier transformations of the impedances. In Sec. VII applications and examples are illustrated, involving different aspect ratios of the cross section, different materials using both AC and DC conductivity models, and different leading and trailing charges displacement from the beam pipe axis.

\section{PHYSICAL MODEL DESCRIPTION}

Let us consider an elliptical cross-section pipe with finite conductivity $\sigma$ and infinite wall thickness, and denote with $a$ the major half-axis of the ellipse and with $b$ the minor one, respectively. Figure 1 shows cross section and longitudinal section of the beam pipe, and the traveling point 

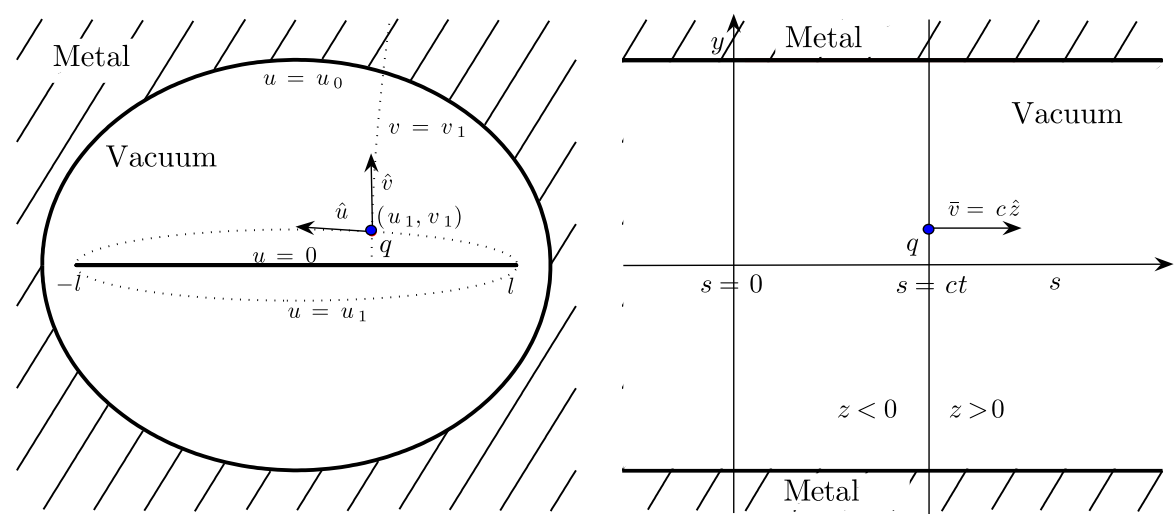

FIG. 1. (Color) The $s-y$ plane view and cross-section plane view.

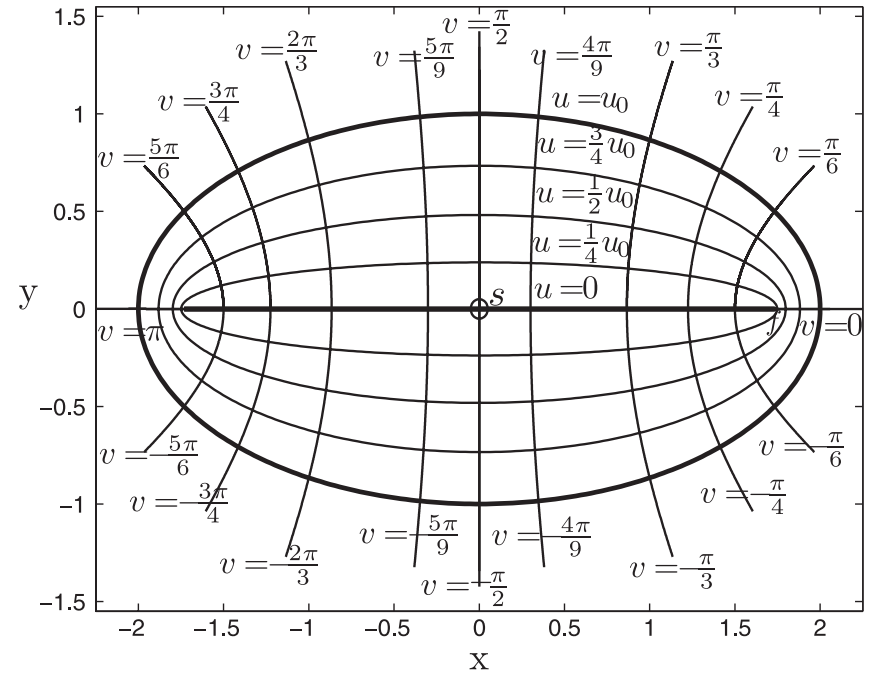

FIG. 2. Elliptic cylindrical $(u, v, s)$ and a Cartesian orthogonal $(x, y, s)$ coordinates system.

charge with the elliptic cylindrical $(u, v, s)$ coordinates system, where the $s$-axis coincides with the pipe axis. The relations between the Cartesian and the elliptical coordinates are given in Appendix A and are illustrated in Fig. 2. The equation $u=u_{0}$ defines the surface separating the vacuum region from the resistive wall. The vacuum region is specified by $u<u_{0}$ while the metal region by $u>$ $u_{0}$. The leading point charge, traveling along the beam pipe, is assumed to be ultrarelativistic, and its longitudinal position is $s=c t$, where $c$ is the velocity of light in vacuum, while its transverse coordinates are $\left(u_{1}, v_{1}\right)$.

\section{FIELDS IN THE VACUUM}

Using the elliptical cylindrical coordinates system $O(u, v, s)$, the Maxwell's equations in the vacuum region can be written as follows, denoting the electric field components with $E_{u}, E_{v}$, and $E_{s}$, and the magnetic field com- ponents $B_{u}, B_{v}$, and $B_{s}$ :

$$
\begin{aligned}
\frac{1}{h^{2}} \frac{\partial h E_{u}}{\partial u}+\frac{1}{h^{2}} \frac{\partial h E_{v}}{\partial v}+\frac{\partial E_{s}}{\partial s} & =\frac{\rho}{\epsilon_{0}}, \\
\frac{1}{h} \frac{\partial E_{s}}{\partial v}-\frac{\partial E_{v}}{\partial s} & =-\frac{\partial B_{u}}{\partial t}, \\
\frac{\partial E_{u}}{\partial s}-\frac{1}{h} \frac{\partial E_{s}}{\partial u} & =-\frac{\partial B_{v}}{\partial t}, \\
\frac{1}{h^{2}} \frac{\partial h E_{v}}{\partial u}-\frac{1}{h^{2}} \frac{\partial h E_{u}}{\partial v} & =-\frac{\partial B_{s}}{\partial t}, \\
\frac{1}{h^{2}} \frac{\partial h B_{u}}{\partial u}+\frac{1}{h^{2}} \frac{\partial h B_{v}}{\partial v}+\frac{\partial B_{s}}{\partial s} & =0, \\
\frac{1}{h} \frac{\partial B_{s}}{\partial v}-\frac{\partial B_{v}}{\partial s} & =\frac{1}{c^{2}} \frac{\partial E_{u}}{\partial t}, \\
\frac{\partial B_{u}}{\partial s}-\frac{1}{h} \frac{\partial B_{s}}{\partial u} & =\frac{1}{c^{2}} \frac{\partial E_{v}}{\partial t}, \\
\frac{1}{h^{2}} \frac{\partial h B_{v}}{\partial u}-\frac{1}{h^{2}} \frac{\partial h B_{u}}{\partial v} & =\frac{1}{c^{2}} \frac{\partial E_{s}}{\partial t}+\mu_{0} J,
\end{aligned}
$$

where $h$ is the metric while the charge and current density are

$$
\begin{aligned}
& \rho=q \frac{\delta(s-c t) \delta\left(u-u_{1}\right) \delta\left(v-v_{1}\right)}{h^{2}} \\
& J=q c \frac{\delta(s-c t) \delta\left(u-u_{1}\right) \delta\left(v-v_{1}\right)}{h^{2}} \hat{s} .
\end{aligned}
$$

In addition, we refer to the coordinate $z=s-c t$, which is the longitudinal displacement from the point charge. Thus it is $z<0$ behind the leading charge and $z>0$ ahead of it. Because of the causality principle, all fields must vanish for $z>0$.

Using the same approach adopted in [4], we write the field vectors $\boldsymbol{E}=\left(E_{u}, E_{v}, E_{z}\right)$ and $\boldsymbol{B}=\left(B_{u}, B_{v}, B_{z}\right)$ in terms of the Fourier transformed vectors $\tilde{\boldsymbol{E}}, \tilde{\boldsymbol{B}}$ on the $z$-axis:

$$
(\boldsymbol{E}, \boldsymbol{B})=\frac{1}{2 \pi} \int_{-\infty}^{+\infty}(\tilde{\boldsymbol{E}}, \tilde{\boldsymbol{B}}) e^{i k z} d k
$$


Substituting Eq. (2a) into Eq. (1a) and Eq. (2b) into Eq. (1h), one obtains the following system of six equations:

$$
\begin{aligned}
\frac{\partial \tilde{E}_{z}}{\partial v}= & c \frac{\partial \tilde{B}_{z}}{\partial u}, \\
\frac{\partial \tilde{E}_{z}}{\partial u}= & -c \frac{\partial \tilde{B}_{z}}{\partial v}, \\
\frac{\partial h \tilde{E}_{u}}{\partial u}-\frac{\partial c h \tilde{B}_{u}}{\partial v}= & \frac{q}{\epsilon_{0}} \delta\left(u-u_{1}\right) \delta\left(v-v_{1}\right)-i k h^{2} \tilde{E}_{z} \\
& +\frac{i}{k} \frac{\partial^{2} \tilde{E}_{z}}{\partial v^{2}} \\
c \frac{\partial h \tilde{B}_{u}}{\partial u}+\frac{\partial h \tilde{E}_{u}}{\partial v}= & -i k h^{2} c \tilde{B}_{z}+\frac{i c}{k} \frac{\partial^{2} \tilde{B}_{z}}{\partial v^{2}}, \\
\tilde{E}_{v}= & -\frac{i}{h k} \frac{\partial \tilde{E}_{z}}{\partial v}-c \tilde{B}_{u}, \\
c \tilde{B}_{v}= & -\frac{i c}{h k} \frac{\partial \tilde{B}_{z}}{\partial v}+\tilde{E}_{u} .
\end{aligned}
$$

Starting from Eqs. (4a) and (4b), and following the approach described in Appendix B, the longitudinal components of the electric and magnetic fields can be written as follows:

$$
\tilde{E}_{z}=A_{0}+\sum_{n=1}^{+\infty} A_{n} \cosh n u \cos n v+\sum_{n=1}^{+\infty} B_{n} \sinh n u \sin n v,
$$

$c \tilde{B}_{z}=B_{0}+\sum_{n=1}^{+\infty} B_{n} \cosh n u \cos n v-\sum_{n=1}^{+\infty} A_{n} \sinh n u \sin n v$,

where $A_{n}$ and $B_{n}$ are constants to be determined.

Starting from Eqs. (4c) and (4d) and using the solutions in (5), the approach described in Appendix $C$ yields the following expressions for the transverse fields $\tilde{E}_{u}$ and $\tilde{B}_{u}$ :

$$
\begin{aligned}
h \tilde{E}_{u}(u, v) & =K_{\tilde{E} u}+S_{\tilde{E} u}^{c}+S_{\tilde{E} u}^{s}+S_{\tilde{E} u}^{q}, \\
h c \tilde{B}_{u}(u, v) & =K_{\tilde{B} u}+S_{\tilde{B} u}^{c}+S_{\tilde{B} u}^{s}+S_{\tilde{B} u}^{q},
\end{aligned}
$$

where denoting with $H(u)$ the Heaviside step function and letting $W=i k\left(a^{2}-b^{2}\right)$, we can write

$$
\begin{aligned}
K_{\tilde{E} u}= & \sum_{n=1}^{+\infty}\left(E_{n}-\frac{n i}{k} B_{n}\right) \cosh n u \sin n v+\sum_{n=1}^{+\infty} F_{n} \sinh n u \cos n v, \\
K_{\tilde{B} u}= & \sum_{n=1}^{+\infty}\left(F_{n}+\frac{n i}{k} A_{n}\right) \cosh n u \sin n v-\sum_{n=1}^{+\infty} E_{n} \sinh n u \cos n v, \\
S_{\tilde{E} u}^{c}= & -A_{0} W \frac{\sinh 2 u}{4}+\frac{A_{1} W}{16}(\sinh u \cos 3 v-\sinh 3 u \cos v)+W \sum_{n=2}^{+\infty} A_{n} \frac{\sinh n u}{8(n+1)} \cos (n+2) v \\
& +W \sum_{n=2}^{+\infty} A_{n} \frac{\sinh n u}{8(n-1)} \cos (n-2) v-W \sum_{n=2}^{+\infty} A_{n}\left[\frac{\sinh (n+2) u}{8(n+1)}+\frac{\sinh (n-2) u}{8(n-1)}\right] \cos n v, \\
S_{\tilde{E} u}^{s}= & +B_{0} W \frac{\sin 2 v}{4}+\frac{B_{1} W}{16}(\cosh u \sin 3 v-\cosh 3 u \sin v)+W \sum_{n=2}^{+\infty} B_{n} \frac{\cosh n u}{8(n+1)} \sin (n+2) v \\
& +W \sum_{n=2}^{+\infty} B_{n} \frac{\cosh n u}{8(n-1)} \sin (n-2) v-W \sum_{n=2}^{+\infty} B_{n}\left[\frac{\cosh (n+2) u}{8(n+1)}+\frac{\cosh (n-2) u}{8(n-1)}\right] \sin n v, \\
S_{\tilde{B} u}^{c}= & -B_{0} W \frac{\sinh 2 u}{4}+\frac{B_{1} W}{16}(\sinh u \cos 3 v-\sinh 3 u \cos v)+W \sum_{n=2}^{+\infty} B_{n} \frac{\sinh n u}{8(n+1)} \cos (n+2) v \\
& +W \sum_{n=2}^{+\infty} B_{n} \frac{\sinh n u}{8(n-1)} \cos (n-2) v-W \sum_{n=2}^{+\infty} B_{n}\left[\frac{\sinh (n+2) u}{8(n+1)}+\frac{\sinh (n-2) u}{8(n-1)}\right] \cos n v, \\
S_{\tilde{B} u}^{s}= & -A_{0} W \frac{\sin 2 v}{4}+\frac{A_{1} W}{16}(\cosh 3 u \sin v-\cosh u \sin 3 v)-W \sum_{n=2}^{+\infty} A_{n} \frac{\cosh n u}{8(n+1)} \sin (n+2) v \\
& -W \sum_{n=2}^{+\infty} A_{n} \frac{\cosh n u}{8(n-1)} \sin (n-2) v+W \sum_{n=2}^{+\infty} A_{n}\left[\frac{\cosh (n+2) u}{8(n+1)}+\frac{\cosh (n-2) u}{8(n-1)}\right] \sin n v,
\end{aligned}
$$




$$
\begin{aligned}
S_{\tilde{E} u}^{q}= & \frac{q}{2 \pi \epsilon_{0}} H\left(u-u_{1}\right)+\sum_{n=1}^{+\infty} \frac{q}{\pi \epsilon_{0}} \cos n v_{1}\left[H\left(u-u_{1}\right) \cosh n u_{1} \cosh n u+H\left(u_{1}-u\right) \sinh n u_{1} \sinh n u\right] \cos n v \\
& -\sum_{n=1}^{+\infty} \frac{q}{\pi \epsilon_{0}} \sin n v_{1}\left[H\left(u-u_{1}\right) \sinh n u_{1} \sinh n u+H\left(u_{1}-u\right) \cosh n u_{1} \cosh n u\right] \sin n v, \\
S_{\tilde{B} u}^{q}= & \sum_{n=1}^{+\infty} \frac{q}{\pi \epsilon_{0}} \sin n v_{1}\left[H\left(u-u_{1}\right) \sinh n u_{1} \cosh n u+H\left(u_{1}-u\right) \cosh n u_{1} \sinh n u\right] \cos n v \\
& +\sum_{n=1}^{+\infty} \frac{q}{\pi \epsilon_{0}} \cos n v_{1}\left[H\left(u-u_{1}\right) \cosh n u_{1} \sinh n u+H\left(u_{1}-u\right) \sinh n u_{1} \cosh n u\right] \sin n v .
\end{aligned}
$$

The field components $\tilde{E}_{v}$ and $\tilde{B}_{v}$ can be directly obtained from Eqs. (4e) and (4f) using the solutions (5) and (6), yielding

$$
\begin{aligned}
h \tilde{E}_{v} & =K_{\tilde{E} v}+S_{\tilde{E} v}^{c}+S_{\tilde{E} v}^{s}+S_{\tilde{E} v}^{q}, \\
h c \tilde{B}_{v} & =K_{\tilde{B} v}+S_{\tilde{B} v}^{c}+S_{\tilde{B} v}^{s}+S_{\tilde{B} v}^{q}
\end{aligned}
$$

where

$$
\begin{aligned}
K_{\tilde{E} v} & =-K_{\tilde{B} u}, \\
S_{\tilde{E} v}^{s} & =-S_{\tilde{B} u}^{s}+\sum_{n=1}^{+\infty} \frac{n i}{k} A_{n} \cosh n u \sin n v, \\
S_{\tilde{E} v}^{c} & =-S_{\tilde{B} u}^{c}-\sum_{n=1}^{+\infty} \frac{n i}{k} B_{n} \sinh n u \cos n v, \\
S_{\tilde{E} v}^{q} & =-S_{\tilde{B} u}^{q}, \\
K_{\tilde{B} v} & =+K_{\tilde{E} u}, \\
S_{\tilde{B} v}^{s} & =+S_{\tilde{E} u}^{s}+\sum_{n=1}^{+\infty} \frac{n i}{k} B_{n} \cosh n u \sin n v, \\
S_{\tilde{B} v}^{c} & =+S_{\tilde{E} u}^{c}+\sum_{n=1}^{+\infty} \frac{n i}{k} A_{n} \sinh n u \cos n v, \\
S_{\tilde{B} v}^{q} & =+S_{\tilde{E} u}^{q} .
\end{aligned}
$$

\section{FIELDS IN THE RESISTIVE WALL}

The constants $A_{n}, B_{n}, E_{n}$, and $F_{n}$ will be determined by imposing the boundary conditions at the surface separating the vacuum from the resistive wall. To this aim we need to calculate the expressions of the fields inside the wall $(u>$ $\left.u_{0}\right)$. In the conductor we assume that

$$
\begin{aligned}
& \rho=0, \\
& \boldsymbol{J}=\sigma \boldsymbol{E},
\end{aligned}
$$

where $\sigma$ is the conductivity of the metal. Furthermore, the Maxwell equations can be written as

$$
\begin{aligned}
\frac{1}{h^{2}} \frac{\partial h E_{u}}{\partial u}+\frac{1}{h^{2}} \frac{\partial h E_{v}}{\partial v}+\frac{\partial E_{s}}{\partial s} & =0, \\
\frac{1}{h} \frac{\partial E_{s}}{\partial v}-\frac{\partial E_{v}}{\partial s} & =-\frac{\partial B_{u}}{\partial t}, \\
\frac{\partial E_{u}}{\partial s}-\frac{1}{h} \frac{\partial E_{s}}{\partial u} & =-\frac{\partial B_{v}}{\partial t}, \\
\frac{1}{h^{2}} \frac{\partial h E_{v}}{\partial u}-\frac{1}{h^{2}} \frac{\partial h E_{u}}{\partial v} & =-\frac{\partial B_{s}}{\partial t}, \\
\frac{1}{h^{2}} \frac{\partial h B_{u}}{\partial u}+\frac{1}{h^{2}} \frac{\partial h B_{v}}{\partial v}+\frac{\partial B_{s}}{\partial s} & =0, \\
\frac{1}{h} \frac{\partial B_{s}}{\partial v}-\frac{\partial B_{v}}{\partial s} & =\frac{1}{c^{2}} \frac{\partial E_{u}}{\partial t}+\mu_{0} \sigma E_{u}, \\
\frac{\partial B_{u}}{\partial s}-\frac{1}{h} \frac{\partial B_{s}}{\partial u} & =\frac{1}{c^{2}} \frac{\partial E_{v}}{\partial t}+\mu_{0} \sigma E_{v}, \\
\frac{1}{h^{2}} \frac{\partial h B_{v}}{\partial u}-\frac{1}{h^{2}} \frac{\partial h B_{u}}{\partial v} & =\frac{1}{c^{2}} \frac{\partial E_{s}}{\partial t}+\mu_{0} \sigma E_{s} .
\end{aligned}
$$

Using the variable $z=s-c t$ and manipulating Eqs. (11) yields equations

$$
\begin{gathered}
\frac{\partial^{2}}{\partial u^{2}} \tilde{E}_{z}+\frac{\partial^{2}}{\partial v^{2}} \tilde{E}_{z}+h^{2} \lambda^{2} \tilde{E}_{z}=0, \\
\frac{\partial^{2}}{\partial u^{2}} c \tilde{B}_{z}+\frac{\partial^{2}}{\partial v^{2}} c \tilde{B}_{z}+h^{2} \lambda^{2} c \tilde{B}_{z}=0, \\
h \tilde{E}_{u}=\frac{i k}{\lambda^{2}} \frac{\partial}{\partial u} \tilde{E}_{z}+\frac{i k}{\lambda^{2}} \frac{\partial}{\partial v} c \tilde{B}_{z}, \\
h \tilde{E}_{v}=\frac{i k}{\lambda^{2}} \frac{\partial}{\partial v} \tilde{E}_{z}-\frac{i k}{\lambda^{2}} \frac{\partial}{\partial u} c \tilde{B}_{z}, \\
\operatorname{ch} \tilde{B}_{u}=-\left[\frac{i k}{\lambda^{2}}+\frac{i}{k}\right] \frac{\partial}{\partial v} \tilde{E}_{z}+\frac{i k}{\lambda^{2}} \frac{\partial}{\partial u} c \tilde{B}_{z}, \\
\operatorname{ch} \tilde{B}_{v}=\left[\frac{i k}{\lambda^{2}}+\frac{i}{k}\right] \frac{\partial}{\partial u} \tilde{E}_{z}+\frac{i k}{\lambda^{2}} \frac{\partial}{\partial v} c \tilde{B}_{z},
\end{gathered}
$$

where $\lambda^{2}=i k Z_{0} \sigma$ and $\lambda$ is chosen with positive imaginary part.

\section{A. Longitudinal fields in the conductor}

Each of Eqs. (12a) and (12b) can be solved by separating the variables $u$ and $v$, that is assuming the unknown is proportional to the product between two functions $U(u)$ 
and $V(v)$. Substituting the product $U(u) V(v)$

$$
\begin{gathered}
\frac{d^{2}}{d v^{2}} V+[a-2 Q \cos 2 v] V=0, \\
\frac{d^{2}}{d u^{2}} U-[a-2 Q \cosh 2 u] U=0,
\end{gathered}
$$

where $Q=l^{2} \lambda^{2} / 4$ and $a$ is a separation constant.

Equations (13a) and (13b) are called the Mathieu angular and radial equations, respectively [10]. On the other hand, the electric and magnetic fields, as well as the function $V(v)$, must be $2 \pi$-periodic in $v$. There is a countable infinity of values of the constant $a$ that allow $2 \pi$-periodic solutions in $v$. Such values are called the Mathieu characteristic numbers (MCNs), and can be calculated with the algorithms in [11]. In particular, for imaginary values of $Q$ (i.e., in the case of DC conductivity), a useful algorithm can be found in [12].

Adopting the standard notation for the MCNs [13], the constants $a_{2 n}$ and $a_{2 n+1}(n \geq 0)$ produce even $\pi$-periodic and $2 \pi$-periodic solutions, respectively, while the constants $b_{2 n} \quad(n \geq 1)$ and $b_{2 n+1} \quad(n \geq 0)$ produce odd $\pi$-periodic and $2 \pi$-periodic solutions, respectively.

Furthermore the possible solutions $V(v)$ can be written as

$$
\begin{aligned}
U_{a_{2 n}}(u, Q) & =\sum_{m=0}^{+\infty}(-1)^{m+n} A_{2 m}^{2 n}\left[J_{m-n}\left(w_{1}\right) H_{m+n}^{(1)}\left(w_{2}\right)+J_{m+n}\left(w_{1}\right) H_{m-n}^{(1)}\left(w_{2}\right)\right], \\
U_{a_{2 n+1}}(u, Q) & =\sum_{m=0}^{+\infty}(-1)^{m+n} A_{2 m+1}^{2 n+1}\left[J_{m-n}\left(w_{1}\right) H_{m+n+1}^{(1)}\left(w_{2}\right)+J_{m+n+1}\left(w_{1}\right) H_{m-n}^{(1)}\left(w_{2}\right)\right], \\
U_{b_{2 n}}(u, Q) & =\sum_{m=1}^{+\infty}(-1)^{m+n} B_{2 m}^{2 n}\left[J_{m-n}\left(w_{1}\right) H_{m+n}^{(1)}\left(w_{2}\right)-J_{m+n}\left(w_{1}\right) H_{m-n}^{(1)}\left(w_{2}\right)\right], \\
U_{b_{2 n+1}}(u, Q) & =\sum_{m=0}^{+\infty}(-1)^{m+n} B_{2 m+1}^{2 n+1}\left[J_{m-n}\left(w_{1}\right) H_{m+n+1}^{(1)}\left(w_{2}\right)-J_{m+n+1}\left(w_{1}\right) H_{m-n}^{(1)}\left(w_{2}\right)\right],
\end{aligned}
$$

where $J_{n}$ is the Bessel function of the first kind of $n$th order, $H_{n}^{(1)}$ is the Hankel function of first kind of $n$th order, $w_{1}=$ $\sqrt{Q} e^{-u}$ and $w_{2}=\sqrt{Q} e^{u}, \sqrt{Q}$ having positive imaginary part.

Being proportional to $U(u) V(v)$, the unknowns $\tilde{E}_{z}$ and $c \tilde{B}_{z}$, can be finally expressed as

$$
\begin{aligned}
\tilde{E}_{z} & =\sum_{n=0}^{+\infty} C_{a_{n}}^{E} U_{a_{n}}(u) \sum_{m=0}^{+\infty} A_{m}^{a_{n}} \cos m v+\sum_{n=1}^{+\infty} D_{b_{n}}^{E} U_{b_{n}}(u) \sum_{m=1}^{+\infty} B_{m}^{b_{n}} \sin m v, \\
c \tilde{B}_{z} & =\sum_{n=0}^{+\infty} C_{a_{n}}^{B} U_{a_{n}}(u) \sum_{m=0}^{+\infty} A_{m}^{a_{n}} \cos m v+\sum_{n=1}^{+\infty} D_{b_{n}}^{B} U_{b_{n}}(u) \sum_{m=1}^{+\infty} B_{m}^{b_{n}} \sin m v,
\end{aligned}
$$

where the constants $C_{a_{n}}^{E}, D_{b_{n}}^{E}, C_{a_{n}}^{B}, D_{b_{n}}^{B}$ are to be determined.

\section{B. Transverse fields in the conductor}

The transverse fields in the conductor can be directly calculated by substituting Eqs. (16a) and (16b) into Eqs. (12c)(12f). As in the following only tangential fields are involved, we give only the expressions for $h \tilde{E}_{v}$ and $\operatorname{ch} \tilde{B}_{v}$ : 


$$
\begin{aligned}
h \tilde{E}_{v}= & \sum_{n=0}^{+\infty}\left[-\frac{i k}{\lambda^{2}} C_{a_{n}}^{E} U_{a_{n}} \sum_{m=1}^{+\infty} m A_{m}^{a_{n}} \sin m v-\frac{i k}{\lambda^{2}} C_{a_{n}}^{B} U_{a_{n}}^{\prime} \sum_{m=0}^{+\infty} A_{m}^{a_{n}} \cos m v\right] \\
& +\sum_{n=1}^{+\infty}\left[\frac{i k}{\lambda^{2}} D_{b_{n}}^{E} U_{b_{n}} \sum_{m=1}^{+\infty} m B_{m}^{b_{n}} \cos m v-\frac{i k}{\lambda^{2}} D_{b_{n}}^{B} U_{b_{n}}^{\prime} \sum_{m=1}^{+\infty} B_{m}^{b_{n}} \sin m v\right] \\
\operatorname{ch} \tilde{B}_{v}= & \sum_{n=0}^{+\infty}\left[\left(\frac{i k}{\lambda^{2}}+\frac{i}{k}\right) C_{a_{n}}^{E} U_{a_{n}}^{\prime} \sum_{m=0}^{+\infty} A_{m}^{a_{n}} \cos m v-\frac{i k}{\lambda^{2}} C_{a_{n}}^{B} U_{a_{n}} \sum_{m=1}^{+\infty} m A_{m}^{a_{n}} \sin m v\right] \\
& +\sum_{n=1}^{+\infty}\left[\left(\frac{i k}{\lambda^{2}}+\frac{i}{k}\right) D_{b_{n}}^{E} U_{b_{n}}^{\prime} \sum_{m=1}^{+\infty} B_{m}^{b_{n}} \sin m v+\frac{i k}{\lambda^{2}} D_{b_{n}}^{B} U_{b_{n}} \sum_{m=1}^{+\infty} m B_{m}^{b_{n}} \cos m v\right] .
\end{aligned}
$$

\section{EVALUATION OF THE CONSTANTS}

The constants involved in the field expressions are determined by satisfying the boundary conditions at $u=u_{0}$, separating the vacuum from the resistive wall.

Precisely, the continuity of $\tilde{E}_{z}, h \tilde{E}_{v}, c \tilde{B}_{z}$, and $h c \tilde{B}_{v}$, the field components tangential to the surface $u=u_{0}$, is imposed. As a result, the constants $A_{2 n}, A_{2 n+1}, B_{2 n}$, and $B_{2 n+1}$ can be calculated by solving through four independent tridiagonal linear systems. More details about the estimation of the linear system can be found in Appendix D. Truncating every tridiagonal linear system at the $(M+$ 1)th order we obtain

$$
\left(\begin{array}{ccccc}
d_{0} & z_{0} & & & \\
s_{0} & d_{1} & z_{1} & & \\
& s_{1} & \ddots & \ddots & \\
& & \ddots & \ddots & z_{M-1} \\
& & & s_{M-1} & d_{M}
\end{array}\right)\left(\begin{array}{c}
X_{0} \\
X_{1} \\
\vdots \\
\vdots \\
X_{M}
\end{array}\right)=\left(\begin{array}{c}
t_{0} \\
t_{1} \\
\vdots \\
\vdots \\
t_{M}
\end{array}\right) .
$$

Since the coefficients on the diagonals are not zero, the system can be easily determined by Gaussian elimination without pivoting. Thus, the following recursive formula can be used to obtain the coefficients:

$$
\begin{gathered}
X_{M}=\frac{T_{M}^{c}}{C_{M}}, \\
X_{n}=\frac{T_{n}^{c}-z_{n} X_{n+1}}{C_{n}},
\end{gathered}
$$

where

$$
\begin{aligned}
& C_{n}= \begin{cases}d_{0} & n=0 \\
d_{n}-\frac{s_{n-1} z_{n-1}}{C_{n-1}} & n=1 \ldots M\end{cases} \\
& D_{n}=\frac{s_{n-1}}{C_{n-1}} \quad n=1 \ldots M \\
& T_{n}^{c}= \begin{cases}t_{0} & n=0 \\
t_{n}-T_{n-1}^{c} D_{n} & n=1 \ldots M .\end{cases}
\end{aligned}
$$

Introducing the quantities $l=\sqrt{a^{2}-b^{2}}, W=i k l^{2}, \lambda^{2}=$ $i k Z_{0} \sigma, R\left(u_{0}\right)=i l \lambda \cosh u_{0}$, the coefficients $s_{n}, d_{n}, z_{n}$, and $t_{n}$ on the diagonals are given by the following relations for each system:

\section{A. System involving the constants $A_{2 n}$}

$$
\begin{aligned}
d_{0}= & -\frac{W}{4} \sinh 2 u_{0}-R\left(u_{0}\right)\left[\frac{i k}{\lambda^{2}}+\frac{i}{k}\right], \\
d_{n}= & -W\left[\frac{\sinh (2 n+2) u_{0}}{(16 n+8) \sinh 2 n u_{0}}+\frac{\cosh (2 n+2) u_{0}}{(16 n+8) \cosh 2 n u_{0}}+\frac{\sinh (2 n-2) u_{0}}{(16 n-8) \sinh 2 n u_{0}}+\frac{\cosh (2 n-2) u_{0}}{(16 n-8) \cosh 2 n u_{0}}\right] \\
& +\frac{2 n i}{k}-\frac{i}{k} R\left(u_{0}\right) \operatorname{coth} 2 n u_{0}-\frac{i k}{\lambda^{2}}\left[R\left(u_{0}\right)\left(\tanh 2 n u_{0}+\operatorname{coth} 2 n u_{0}\right)-4 n\right] \quad n \geq 1, \\
z_{0}= & \frac{W}{8} \sinh 2 u_{0}, \quad z_{n}=\frac{W}{16 n+8}\left[\frac{\sinh (2 n+2) u_{0}}{\sinh 2 n u_{0}}+\frac{\cosh (2 n+2) u_{0}}{\cosh 2 n u_{0}}\right] \quad n \geq 1, \\
s_{0}= & \frac{W}{4} \frac{1}{\cosh 2 u_{0}}, \quad s_{n}=\frac{W}{16 n+8}\left[\frac{\sinh 2 n u_{0}}{\sinh (2 n+2) u_{0}}+\frac{\cosh 2 n u_{0}}{\cosh (2 n+2) u_{0}}\right] \quad n \geq 1, \\
t_{0}= & -\frac{q}{2 \pi \epsilon_{0}}, \quad t_{n}=\frac{q}{\pi \epsilon_{0}} \cos 2 n v_{1} \cosh 2 n u_{1}\left[\tanh 2 n u_{0}-\operatorname{coth} 2 n u_{0}\right] \quad n \geq 1 .
\end{aligned}
$$




\section{B. System involving the constants $A_{2 n+1}$}

$$
\begin{aligned}
d_{0}= & -\frac{W}{16}\left[\frac{\sinh 3 u_{0}}{\sinh u_{0}}+\frac{\cosh 3 u_{0}}{\cosh u_{0}}\right]+\frac{i}{k}-\frac{i}{k} R\left(u_{0}\right) \operatorname{coth} u_{0}-\frac{i k}{\lambda^{2}}\left[R\left(u_{0}\right)\left(\tanh u_{0}+\operatorname{coth} u_{0}\right)-2\right], \\
d_{n}= & -\frac{W}{16(n+1)}\left[\frac{\sinh (2 n+3) u_{0}}{\sinh (2 n+1) u_{0}}+\frac{\cosh (2 n+3) u_{0}}{\cosh (2 n+1) u_{0}}\right]-\frac{W}{16 n}\left[\frac{\sinh (2 n-1) u_{0}}{\sinh (2 n+1) u_{0}}+\frac{\cosh (2 n-1) u_{0}}{\cosh (2 n+1) u_{0}}\right] \\
& +\frac{(2 n+1) i}{k}-\frac{i}{k} \frac{R\left(u_{0}\right)}{\tanh (2 n+1) u_{0}}-\frac{i k}{\lambda^{2}}\left[R\left(u_{0}\right)\left(\tanh (2 n+1) u_{0}+\operatorname{coth}(2 n+1) u_{0}\right)-2(2 n+1)\right] \quad n \geq 1, \\
z_{n-1}= & \frac{W}{16 n}\left[\frac{\sinh (2 n+1) u_{0}}{\sinh (2 n-1) u_{0}}+\frac{\cosh (2 n+1) u_{0}}{\cosh (2 n-1) u_{0}}\right] \quad n \geq 1, \\
s_{n-1}= & \frac{W}{16 n}\left[\frac{\sinh (2 n-1) u_{0}}{\sinh (2 n+1) u_{0}}+\frac{\cosh (2 n-1) u_{0}}{\cosh (2 n+1) u_{0}}\right] \quad n \geq 1, \\
t_{n-1}= & \frac{q}{\pi \epsilon_{0}} \cos (2 n-1) v_{1} \cosh (2 n-1) u_{1}\left[\tanh (2 n-1) u_{0}-\operatorname{coth}(2 n-1) u_{0}\right] \quad n \geq 1 .
\end{aligned}
$$

\section{System involving the constants $B_{2 n}$}

$$
\begin{aligned}
d_{0}= & +\frac{W}{4} \sinh 2 u_{0}+R\left(u_{0}\right) \frac{i k}{\lambda^{2}}, \\
d_{n}= & +W\left[\frac{\sinh (2 n+2) u_{0}}{(16 n+8) \sinh 2 n u_{0}}+\frac{\cosh (2 n+2) u_{0}}{(16 n+8) \cosh 2 n u_{0}}+\frac{\sinh (2 n-2) u_{0}}{(16 n-8) \sinh 2 n u_{0}}+\frac{\cosh (2 n-2) u_{0}}{(16 n-8) \cosh 2 n u_{0}}\right] \\
& -\frac{2 n i}{k}+\frac{i}{k} R\left(u_{0}\right) \tanh 2 n u_{0}+\frac{i k}{\lambda^{2}}\left[R\left(u_{0}\right)\left(\tanh 2 n u_{0}+\operatorname{coth} 2 n u_{0}\right)-4 n\right] \quad n \geq 1, \quad z_{0}=-\frac{W}{8} \sinh 2 u_{0}, \\
z_{n}= & -\frac{W}{16 n+8}\left[\frac{\sinh (2 n+2) u_{0}}{\sinh 2 n u_{0}}+\frac{\cosh (2 n+2) u_{0}}{\cosh 2 n u_{0}}\right] \quad n \geq 1, \quad s_{0}=-\frac{W}{4} \frac{1}{\cosh 2 u_{0}}, \\
s_{n}= & -\frac{W}{16 n+8}\left[\frac{\sinh 2 n u_{0}}{\sinh (2 n+2) u_{0}}+\frac{\cosh 2 n u_{0}}{\cosh (2 n+2) u_{0}}\right] \quad n \geq 1, \quad t_{0}=0, \\
t_{n}= & \frac{q}{\pi \epsilon_{0}} \sin 2 n v_{1} \sinh 2 n u_{1}\left[\operatorname{coth} 2 n u_{0}-\tanh 2 n u_{0}\right] \quad n \geq 1 .
\end{aligned}
$$

\section{System involving the constants $B_{2 n+1}$}

$$
\begin{aligned}
d_{0}= & +\frac{W}{16}\left[\frac{\sinh 3 u_{0}}{\sinh u_{0}}+\frac{\cosh 3 u_{0}}{\cosh u_{0}}\right]-\frac{i}{k}+\frac{i}{k} R\left(u_{0}\right) \tanh u_{0}+\frac{i k}{\lambda^{2}}\left[R\left(u_{0}\right)\left(\tanh u_{0}+\operatorname{coth} u_{0}\right)-2\right], \\
d_{n}= & +\frac{W}{16(n+1)}\left[\frac{\sinh (2 n+3) u_{0}}{\sinh (2 n+1) u_{0}}+\frac{\cosh (2 n+3) u_{0}}{\cosh (2 n+1) u_{0}}\right]+\frac{W}{16 n}\left[\frac{\sinh (2 n-1) u_{0}}{\sinh (2 n+1) u_{0}}+\frac{\cosh (2 n-1) u_{0}}{\cosh (2 n+1) u_{0}}\right]-\frac{(2 n+1) i}{k} \\
& +\frac{i}{k} R\left(u_{0}\right) \tanh (2 n+1) u_{0}+\frac{i k}{\lambda^{2}}\left[R\left(u_{0}\right)\left(\tanh (2 n+1) u_{0}+\operatorname{coth}(2 n+1) u_{0}\right)-2(2 n+1)\right] \quad n \geq 1, \\
z_{n-1}= & -\frac{W}{16 n}\left[\frac{\sinh (2 n+1) u_{0}}{\sinh (2 n-1) u_{0}}+\frac{\cosh (2 n+1) u_{0}}{\cosh (2 n-1) u_{0}}\right] \quad n \geq 1, \\
s_{n-1}= & -\frac{W}{16 n}\left[\frac{\sinh (2 n-1) u_{0}}{\sinh (2 n+1) u_{0}}+\frac{\cosh (2 n-1) u_{0}}{\cosh (2 n+1) u_{0}}\right] \quad n \geq 1, \quad n \geq 1 . \\
t_{n-1}= & \frac{q}{\pi \epsilon_{0}} \sin (2 n-1) v_{1} \sinh (2 n-1) u_{1}\left[\operatorname{coth}(2 n-1) u_{0}-\tanh (2 n-1) u_{0}\right] \quad n \geq
\end{aligned}
$$




\section{SHORT-RANGE WAKE FUNCTIONS}

Let us consider an ultrarelativistic trailing charge $q_{t}$ traveling with a longitudinal displacement $z$ from the leading charge $q$; its transverse coordinates are $(u, v)$. The Lorentz force experienced by the trailing charge due to the leading charge is

$$
\boldsymbol{F}=q_{t}(\boldsymbol{E}+\hat{z} \times c \boldsymbol{B}) .
$$

The longitudinal and transverse components of $\boldsymbol{F}$ are

$$
\begin{aligned}
& F_{L}=q_{t} E_{z}, \\
& \boldsymbol{F}_{\boldsymbol{T}}=q_{t}\left[\hat{u}\left(E_{u}-c B_{v}\right)+\hat{v}\left(E_{v}+c B_{u}\right)\right],
\end{aligned}
$$

where the fields $E_{z}, E_{u}, E_{v}, B_{u}$, and $B_{v}$ are evaluated in $(u, v, z)$ and depend on the position $\left(u_{1}, v_{1}, 0\right)$ of the leading charge $q$. Using Eqs. (4e) and (4f); we can write

$$
\begin{aligned}
& \tilde{E}_{v}+c \tilde{B}_{u}=-\frac{i}{h k} \frac{\partial \tilde{E}_{z}}{\partial v} \\
& \tilde{E}_{u}-c \tilde{B}_{v}=+\frac{i}{h k} \frac{\partial c \tilde{B}_{z}}{\partial v} .
\end{aligned}
$$

Thus, the force depends only on the longitudinal components $E_{z}$ and $B_{z}$.

The wake function per unit of length is defined by $\boldsymbol{W}=$ $\boldsymbol{F} / q q_{t}$, and is related to the impedance through the following Fourier transformation:

$$
\boldsymbol{Z}(u, v, k)=\frac{1}{c} \int_{-\infty}^{+\infty} \boldsymbol{W}(z, u, v) e^{-i k z} d z .
$$

Consequently, the longitudinal impedance per unit of length is given by $1 / q c$ times the expression of $\tilde{E}_{z}(k)$ in Eq. (5a):

$$
\begin{aligned}
Z_{L}(u, v, k)= & \sum_{n=0}^{+\infty} \frac{A_{n}}{q c} \cosh n u \cos n v+\sum_{n=1}^{+\infty} \frac{B_{n}}{q c} \\
& \times \sinh n u \sin n v
\end{aligned}
$$

while the two components $Z_{u}$ and $Z_{v}$ of the transverse impedance $\boldsymbol{Z}_{T}=Z_{u} \hat{u}+Z_{v} \hat{v}$ are calculated using Eqs. (27b) and (28):

$$
\begin{aligned}
Z_{u}(u, v, k)= & -\frac{i}{q \operatorname{ch} k} \sum_{n=1}^{+\infty} n\left(A_{n} \sinh n u \cos n v\right. \\
& \left.+B_{n} \cosh n u \sin n v\right) \\
Z_{v}(u, v, k)= & +\frac{i}{q \operatorname{chk}} \sum_{n=1}^{+\infty} n\left(A_{n} \cosh n u \sin n v\right. \\
& \left.-B_{n} \sinh n u \cos n v\right)
\end{aligned}
$$

Thus, the wake functions are obtained by a numerical inverse Fourier transformation of the impedance. The fields vanish for $z>0$ and since the real part of the impedance drops more quickly for large values of $k$ than the imaginary part it seems to be more convenient to use the cosine inverse transformation:

$$
\begin{gathered}
W_{L}=\frac{2 c}{\pi} \int_{0}^{+\infty} \Re\left(Z_{L}\right) \cos (k z) d k \\
\boldsymbol{W}_{T}=\frac{2 c}{\pi} \int_{0}^{+\infty}\left[\Re\left(Z_{v}\right) \hat{v}+\Re\left(Z_{u}\right) \hat{u}\right] \cos (k z) d k .
\end{gathered}
$$

\section{EXAMPLES AND RESULTS}

In this section the impedances and the short wake functions are calculated for several cases, such as cross sections with different aspect ratio values $a / b$ and two different kinds of conductors, aluminum, and copper with DC and $\mathrm{AC}$ conductivity values. The conductivity $\sigma$ and the relaxation time $\tau$ used for aluminum and copper are listed in Table I. The relative displacement between the leading and the trailing charge is also considered. Basically, the tridiagonal linear system in Eq. (18) must be truncated and when we consider large values for the aspect ratio $a / b$, more sine and cosine components are needed to represent the fields accurately. A particular case is when both charges, leading and trailing, travel along on-axis in the vacuum chamber. In this case, only the $A$ even subsystem is excited, and the longitudinal impedance is then simplified as

$$
Z_{L}(u, v, k)=\sum_{n=0}^{+\infty} \frac{(-1)^{n}}{q c} A_{2 n}
$$

In a small region around the axis, the longitudinal wake function remains approximately constant while the transverse wake function depends linearly on the displacements of the leading and the trailing charges. This effect is shown in Fig. 3. The longitudinal wake function has been calculated at $z=0$ with different transverse positions of both the leading and the trailing charges from the axis (offset). Figures 3(a) and 3(b) show these values normalized to the value of the wake function on axis. For the transverse wake function, the maximum values divided by the offset of the charges have been calculated, and Figs. 3(c) and 3(d) show these values normalized with respect to the limit of the wake function for the offset approaching zero. In this way it is shown that, if the offset is sufficiently small, the longitudinal wake function is approximately equal to the value on axis, while the transverse wake function can be considered as a linear function of the offset. For this

TABLE I. Conductivity and electron relaxation time for aluminum and copper.

\begin{tabular}{lcc}
\hline \hline & \multicolumn{1}{c}{$\sigma$} & $\tau$ \\
\hline Aluminum & $4.2281 \times 10^{7} \mathrm{Sm}^{-1}$ & $8.0055 \times 10^{-15} \mathrm{~s}$ \\
Copper & $6.4534 \times 10^{7} \mathrm{Sm}^{-1}$ & $2.7019 \times 10^{-14} \mathrm{~s}$ \\
\hline \hline
\end{tabular}




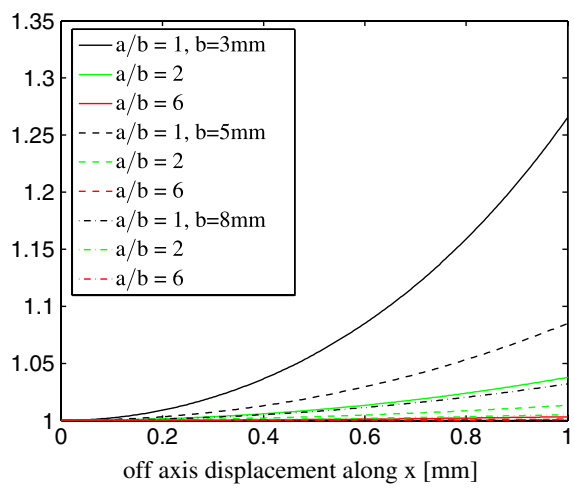

(a) $W_{z}\left(x, x_{1}, z=0\right) / W_{z}\left(x=0, x_{1}=0, z=0\right)$ vs

$$
x=x_{1} \text {. }
$$

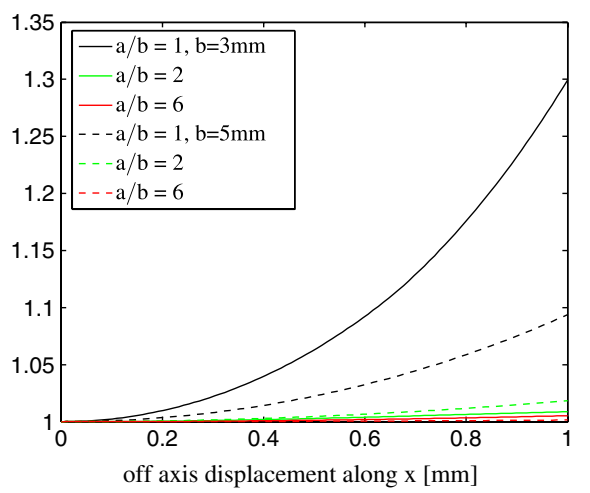

(c) $\max _{z}\left|W_{x}\left(x, x_{1}\right) / x\right| / \max _{z}\left|W_{x}\left(x=\epsilon, x_{1}=\epsilon\right) / \epsilon\right| \mathrm{vs}$ $x=x_{1}, \epsilon<<1 \mu \mathrm{m}$

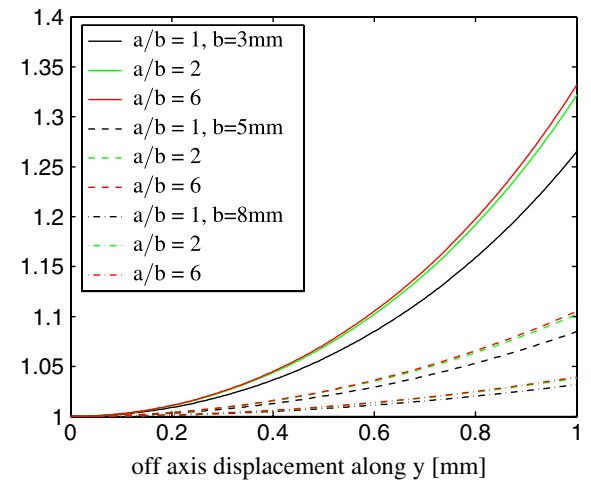

(b) $W_{z}\left(y, y_{1}, z=0\right) / W_{z}\left(y=0, y_{1}=0, z=0\right)$ vs

$$
x=x_{1} .
$$

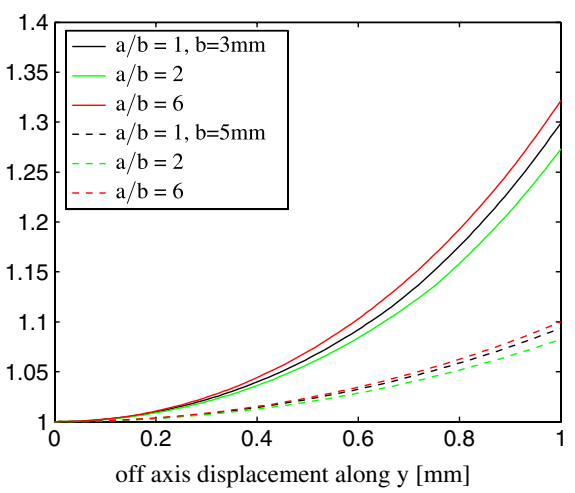

(d) $\max _{z}\left|W_{y}\left(y, y_{1}\right) / y\right| / \max _{z}\left|W_{y}\left(y=\epsilon, y_{1}=\epsilon\right) / \epsilon\right|$ vs $y=y_{1}, \epsilon<<1 \mu \mathrm{m}$.

FIG. 3. (Color) Growth of the longitudinal wake functions and nonlinearity with the offset of the transverse wake functions depending on the offset of the leading and trailing charges.

reason, whenever the offset is small, transverse wake functions are expressed per unit of length of transverse displacement in $\mathrm{V} / \mathrm{pC} / \mathrm{m}^{2}$.

In the following examples we applied our method using vacuum chambers having the short half-axis length $b=$ $3 \mathrm{~mm}$. The reason is that this value is equal to the half gap for the undulator vacuum chamber of the FERMI@Elettra FEL project [14]. Nevertheless to better compare our plots with the results obtained with the boundary element method by Yokoya, Fig. 6 has been obtained using $b=$ $1 \mathrm{~cm}$, the DC conductivity model, and the same aspect ratio values presented in [9].

Figure 4 shows the longitudinal impedance with the AC conductivity model for copper and aluminum, as a function of the wave number $k$ and for several values of the aspect ratio $a / b$.

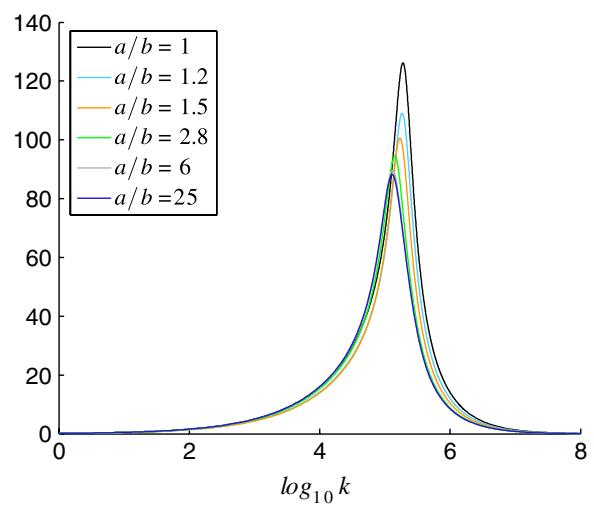

(a) $\left|Z_{L}\right|, \Omega / \mathrm{m}$, aluminum, semi-minor axis $b=3 \mathrm{~mm}$.

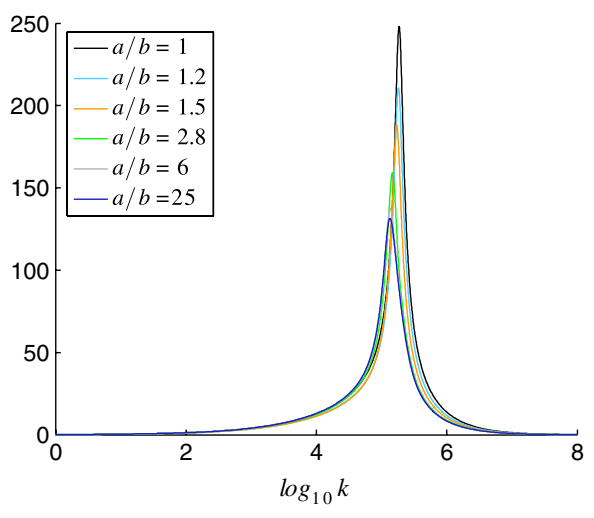

(b) $\left|Z_{L}\right|, \Omega / \mathrm{m}$, copper, semi-minor axis $b=3 \mathrm{~mm}$

FIG. 4. (Color) Longitudinal impedance vs $\log _{10} k\left(k\right.$ in $\left.\mathrm{m}^{-1}\right)$ obtained using the AC conductivity model for aluminum (a) and copper (b), semiaxis $b=3 \mathrm{~mm}$, and with several aspect ratio values $a / b$. 


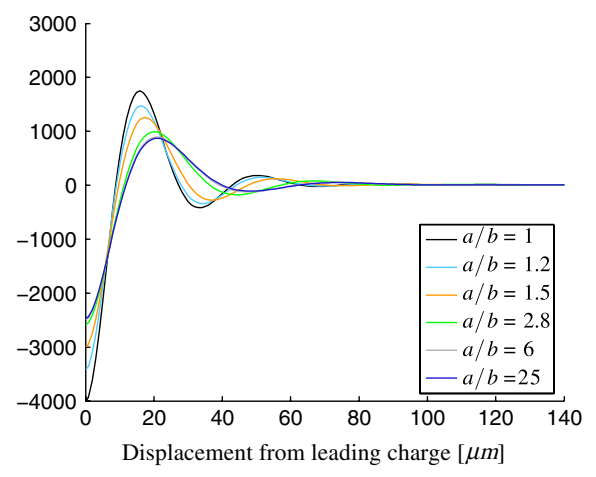

(a) $W_{L}, \mathrm{~V} / \mathrm{pC} / \mathrm{m}, \mathrm{Al}$, with $\mathrm{AC}$ conductivity model.

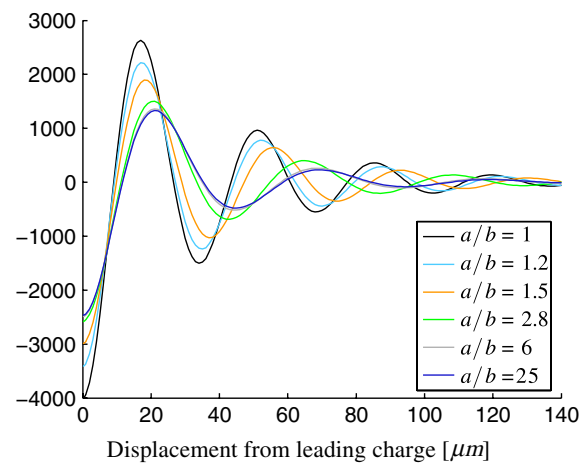

(b) $W_{L}, \mathrm{~V} / \mathrm{pC} / \mathrm{m}, \mathrm{Cu}$, with $\mathrm{AC}$ conductivity model.

FIG. 5. (Color) Dependence of longitudinal wake functions on the longitudinal displacement behind the leading charge. Semiminor axis $b=3 \mathrm{~mm}$, AC conductivity models for aluminum (a) and copper (b), and several aspect ratio values $a / b$.

Figure 5 shows the longitudinal wake functions for a vacuum chamber with semiminor axis $b=3 \mathrm{~mm}$ and several aspect ratio values $a / b$, as a function of the longitudinal displacements behind the leading charge. It is worthwhile noting that the longitudinal wake functions reduce to those of the circular case when $a / b=1$ [7]

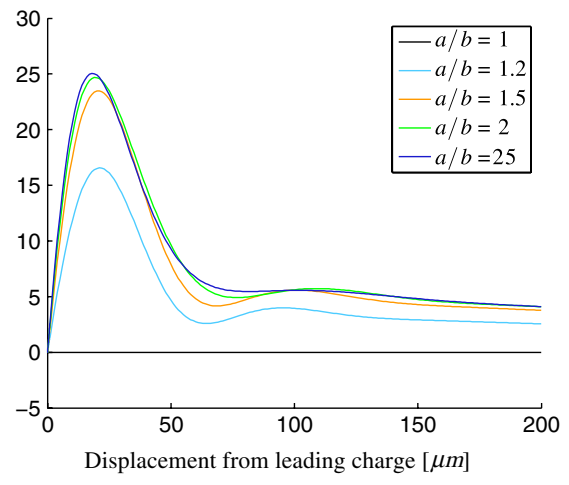

(a) $\partial W_{y} / \partial y, \mathrm{~V} / \mathrm{pC} / \mathrm{m}^{2}$

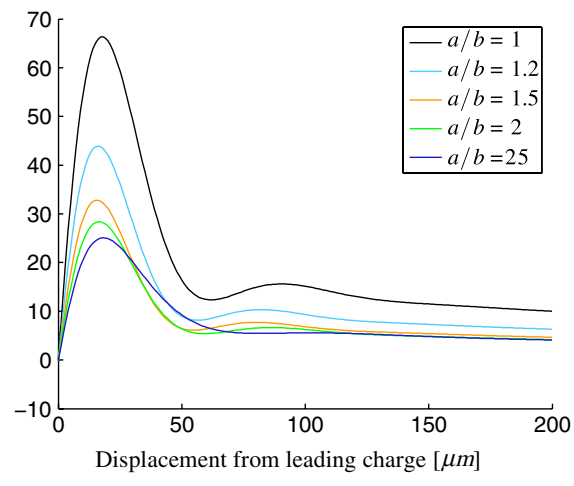

(c) $\partial W_{x} / \partial x, \mathrm{~V} / \mathrm{pC} / \mathrm{m}^{2}$. and to those of the parallel plates when $a / b \gg 1$ [8], respectively.

Figure 6 shows the transverse wake functions obtained using DC conductivity model for copper and a semiaxis $b=10 \mathrm{~mm}$. It is worthy to mention that in the figure the conductivity used is $5.3 \times 10^{7} \mathrm{Sm}^{-1}$ and that the results obtained reduce correctly to the circular case [4] and to the

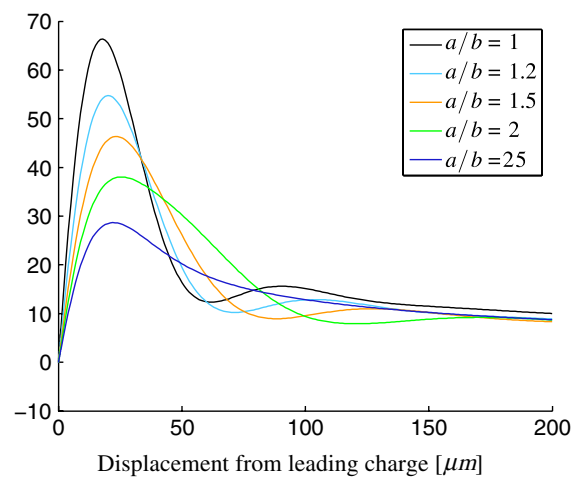

(b) $\partial W_{y} / \partial y_{1}, \mathrm{~V} / \mathrm{pC} / \mathrm{m}^{2}$

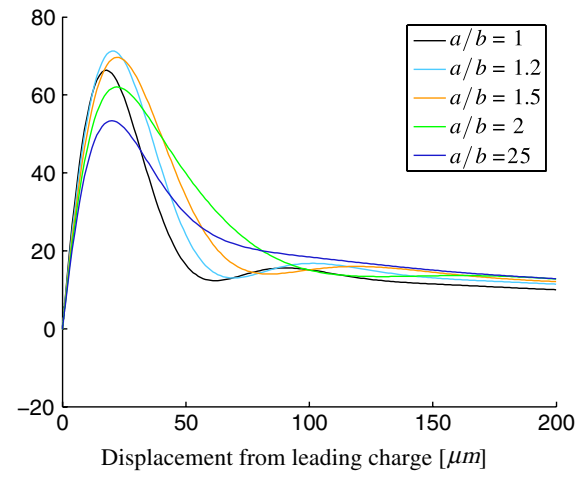

(d) $\partial W_{y} / \partial y+\partial W_{y} / \partial y_{1}, \mathrm{~V} / \mathrm{pC} / \mathrm{m}^{2}$, due to the leading and trailing charghe with the same y-offset.

FIG. 6. (Color) Transverse wake functions in the case where both charges are close to the vacuum chamber axis. The vacuum chamber has short half-axis of length $b=1 \mathrm{~cm}$, the metal wall is characterized by a conductivity $\sigma=5.3 \times 10^{7} \mathrm{Sm}^{-1}$, and the DC conductivity model has been used. 


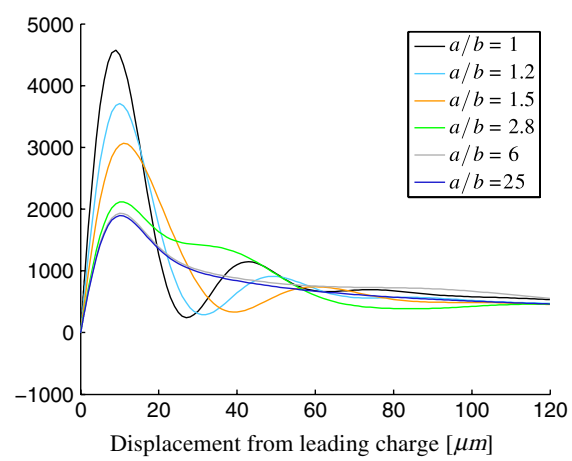

(a) $\partial W_{y} / \partial y_{1}, \mathrm{~V} / \mathrm{pC} / \mathrm{m}^{2}$, the leading charge off axis and the trailing charge on-axis.

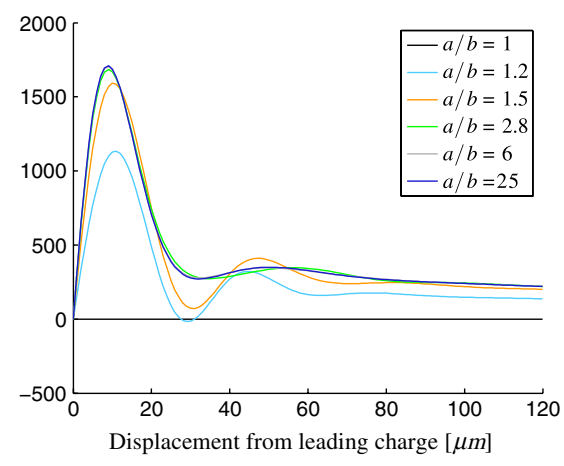

(c) $\partial W_{y} / \partial y, \mathrm{~V} / \mathrm{pC} / \mathrm{m}^{2}$, the trailing charge off axis and the leading charge on-axis.

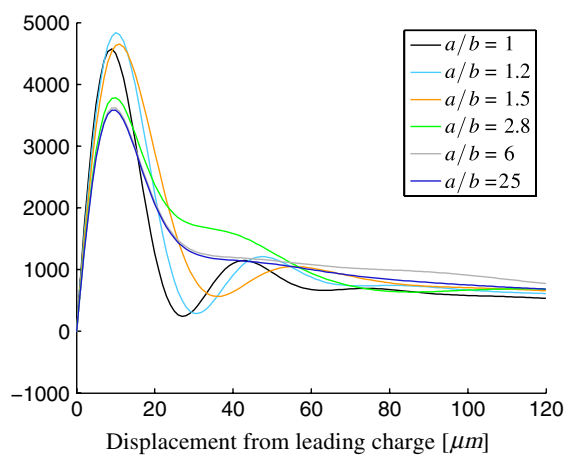

(e) $\partial W_{y} / \partial y_{1}+\partial W_{y} / \partial y, \mathrm{~V} / \mathrm{pC} / \mathrm{m}^{2}$, the leading and the trailing charges off-axis.

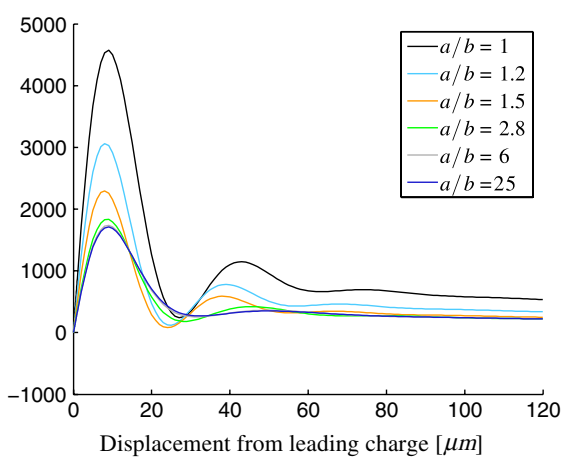

(b) $\partial W_{x} / \partial x_{1}, \mathrm{~V} / \mathrm{pC} / \mathrm{m}^{2}$, the leading charge off axis and the trailing charge on-axis.

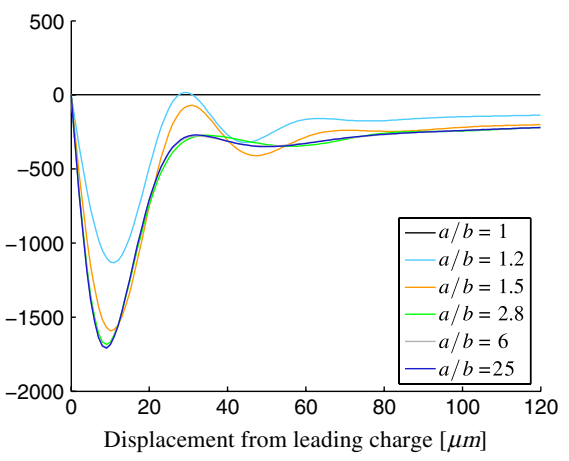

(d) $\partial W_{x} / \partial x, \mathrm{~V} / \mathrm{pC} / \mathrm{m}^{2}$, the trailing charge off axis and the leading charge on-axis.

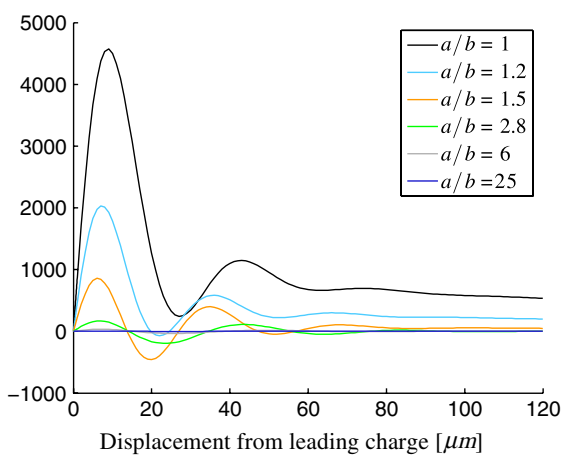

(f) $\partial W_{x} / \partial x_{1}+\partial W_{x} / \partial x, \mathrm{~V} / \mathrm{pC} / \mathrm{m}^{2}$, the leading and the trailing charges off-axis.

FIG. 7. (Color) Dependence of transverse wake functions on the longitudinal displacement behind the leading charge in a small region around the vacuum chamber axis. Semiminor axis $b=3 \mathrm{~mm}$ and several values of the aspect ratio $a / b$ are considered. AC conductivity model for aluminum is used.

parallel plates case [5]. Furthermore, the results are in good agreement with the solutions proposed in [9].

Figure 7 shows the transverse wake functions as functions of the longitudinal displacement behind the leading charge, for aluminum. Here we considered three different relative transverse positions between the leading and the trailing charges: both charges with a $y$ offset, the leading charge off-axis and the trailing charge on axis and vice versa. The semiminor axis $b$ is $3 \mathrm{~mm}$ and the more signifi- cant case of AC conductivity model is considered. Figure 7(f) shows that $\frac{\partial}{\partial x} W_{x}+\frac{\partial}{\partial x_{1}} W_{x}$ vanishes for large values of $a / b$, when the ellipse approaches the parallel plates limit. When an ultrashort bunch is considered, then the transverse wake with the leading and the trailing charges off axis with the same offset should be considered. Figures 7(e) and 7(f) represent this case. As explained in [15], the transverse wake forces near the axis of a bisymmetric pipe (the elliptical pipe has mirror symmetry in both 
$x$ and $y$ ) have the property $\frac{\partial W_{y}}{\partial y}=-\frac{\partial W_{x}}{\partial x}$. This explains why Fig. 7(d) is the mirror of Fig. 7(c).

Once the wake function is known, the energy change per unit length (in $\mathrm{eV} / \mathrm{m}$ ) induced within a particle bunch is obtained from the convolution:

$$
\Delta E(z)=-e Q \int_{-\infty}^{z} W_{L}\left(z-z^{\prime}\right) \rho\left(z^{\prime}\right) d z^{\prime}
$$

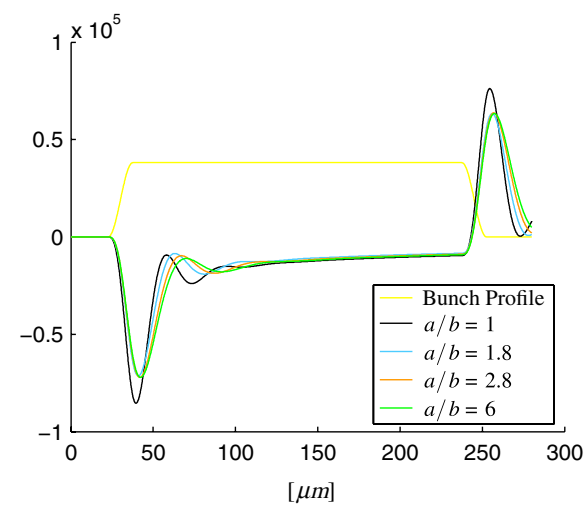

(a) $\Delta E, \mathrm{eV} / \mathrm{m}$, flat length $200 \mu \mathrm{m}$, rise length $15 \mu \mathrm{m}$, aluminum.

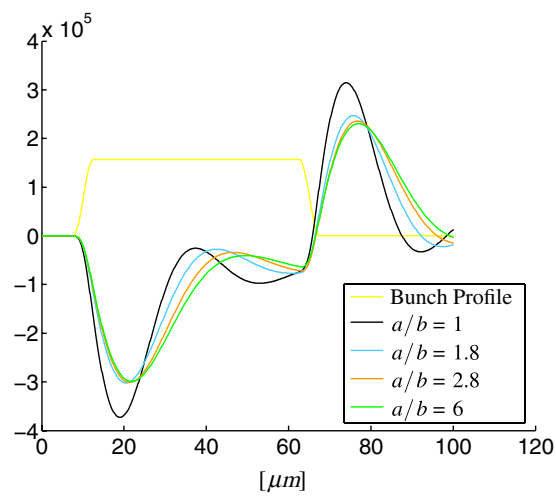

(c) $\Delta E, \mathrm{eV} / \mathrm{m}$, flat length $50 \mu \mathrm{m}$, rise length $5 \mu \mathrm{m}$, aluminum.

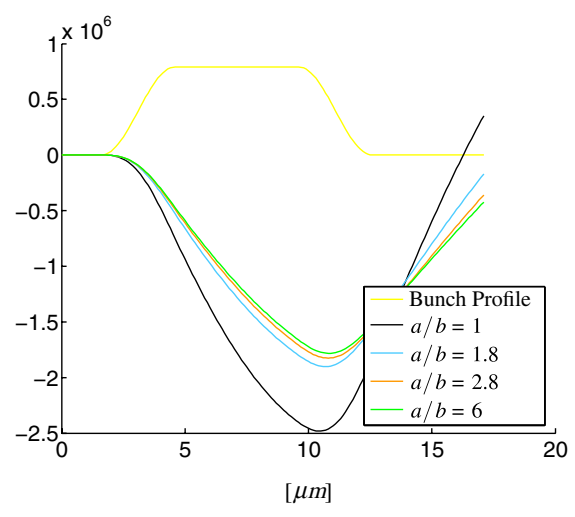

(e) $\Delta E, \mathrm{eV} / \mathrm{m}$, flat length $5 \mu \mathrm{m}$, rise length $3 \mu \mathrm{m}$, aluminum. where $e$ is the electron charge and $Q$ is the total bunch charge with longitudinal charge distribution $\rho(z)$ expressed in $\mathrm{m}^{-1}$. Figure 8 shows the convolutions obtained with the wake functions and flattop bunches of charge equal to $1 \mathrm{nC}$ and different lengths.

As for the longitudinal case, once the transverse wake function is known, the transverse kick for unit length and for transverse offset (in $\mathrm{rad} / \mathrm{m}^{2}$ ) received by the particles in

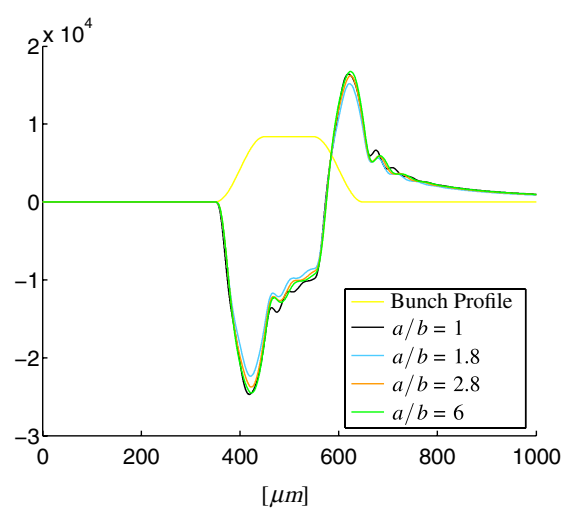

(b) $\Delta E, e V / m$, flat length $200 \mu \mathrm{m}$, rise length $15 \mu \mathrm{m}$, copper.

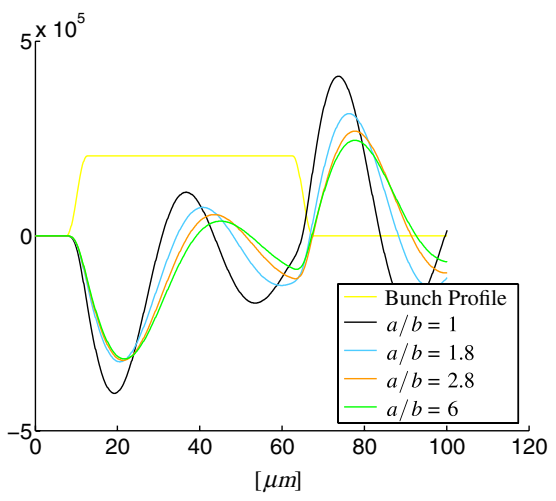

(d) $\Delta E, \mathrm{eV} / \mathrm{m}$, flat length $50 \mu \mathrm{m}$, rise length $5 \mu \mathrm{m}$, copper.

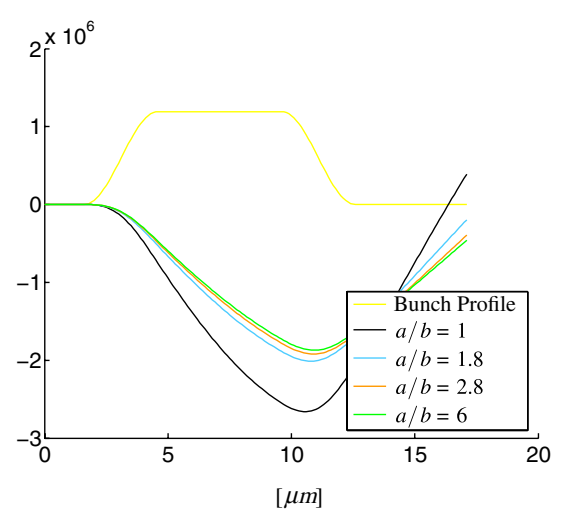

(f) $\Delta E, \mathrm{eV} / \mathrm{m}$, flat length $5 \mu \mathrm{m}$, rise length $3 \mu \mathrm{m}$, copper.

FIG. 8. (Color) Energy variation per unit length induced within flattop bunches with different lengths and charge of $1 \mathrm{nC}$. Semiminor axis $b=3 \mathrm{~mm}$, AC conductivity model, and several aspect ratio values $a / b$ are used. 


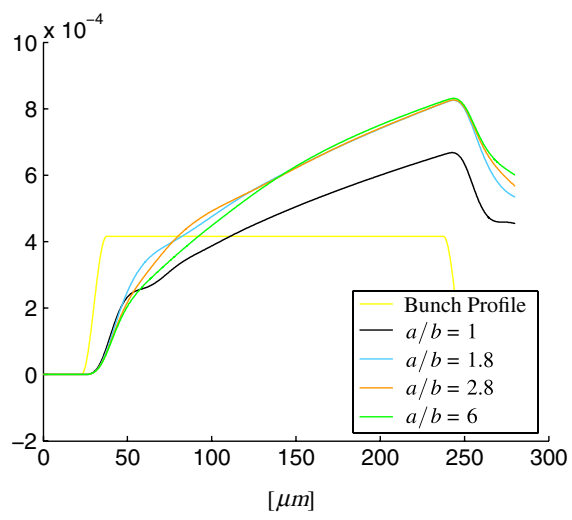

(a) $k_{y}, \mathrm{rad} / \mathrm{m}^{2}$, flat length $200 \mu \mathrm{m}$, rise length $15 \mu \mathrm{m}$, aluminum.

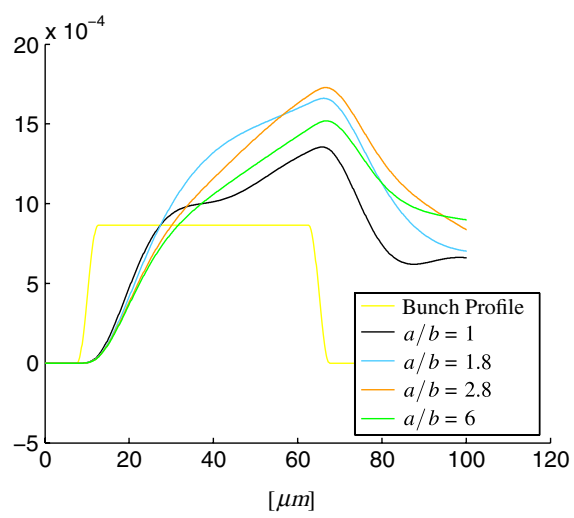

(c) $k_{y}, \mathrm{rad} / \mathrm{m}^{2}$, flat length $50 \mu \mathrm{m}$, rise length $5 \mu \mathrm{m}$, aluminum.

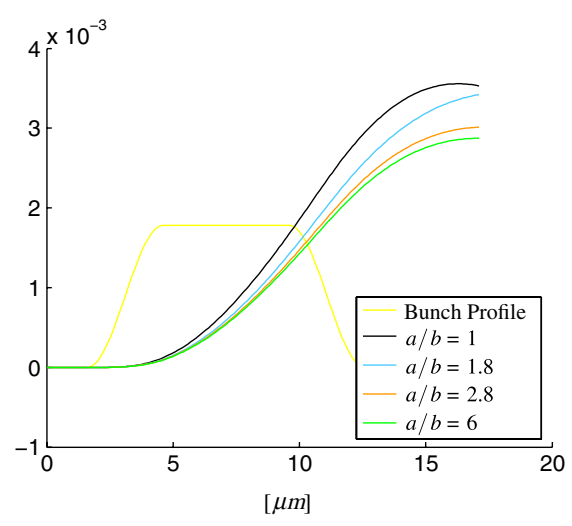

(e) $k_{y}, \mathrm{rad} / \mathrm{m}^{2}$, flat length $5 \mu \mathrm{m}$, rise length $3 \mu \mathrm{m}$, aluminum.

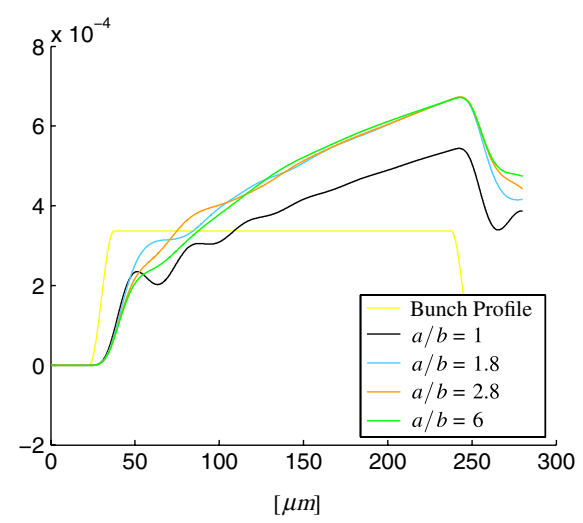

(b) $k_{y}, \mathrm{rad} / \mathrm{m}^{2}$, flat length $200 \mu \mathrm{m}$, rise length $15 \mu \mathrm{m}$, copper.

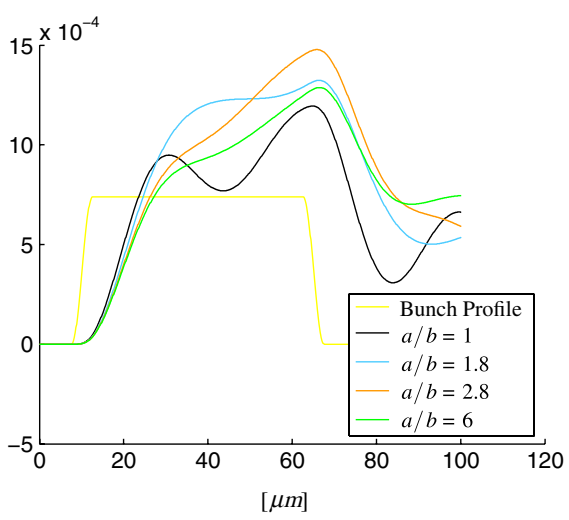

(d) $k_{y}, \mathrm{rad} / \mathrm{m}^{2}$, flat length $50 \mu \mathrm{m}$, rise length $5 \mu \mathrm{m}$, copper.

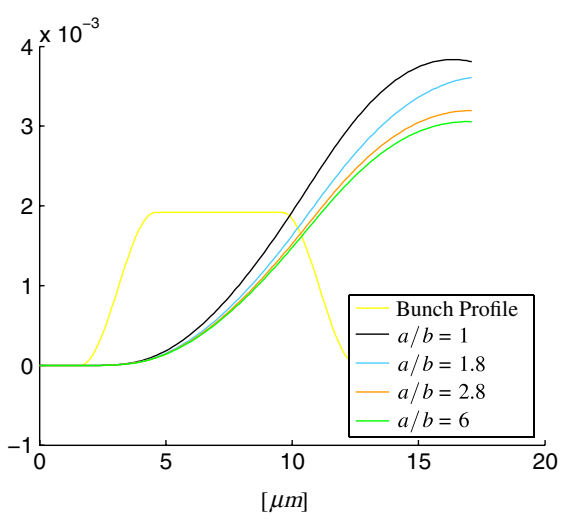

(f) $k_{y}, \mathrm{rad} / \mathrm{m}^{2}$, flat length $5 \mu \mathrm{m}$, rise length $3 \mu \mathrm{m}$, copper.

FIG. 9. (Color) Transversal kick per unit length and off-axis displacement, along the short axis direction, induced within flattop bunches with different lengths and charge $1 \mathrm{nC}$ at $1.2 \mathrm{GeV}$. Semiminor axis $b=3 \mathrm{~mm}$, AC conductivity model, and several aspect ratio values $a / b$ are used.

the vertical plane within the bunch is obtained from the following convolution:

$$
k_{y}(z)=\frac{e Q}{E_{0}} \int_{-\infty}^{z} W_{y}\left(z-z^{\prime}\right) \rho\left(z^{\prime}\right) d z^{\prime},
$$

where $e$ is the electron charge, $Q$ is the total bunch charge with longitudinal bunch distribution $\rho(z)$, and $E_{0}$ is the bunch mean energy. Here only the vertical plane is considered, because for aspect ratio values $a / b>3$ the effect of the transverse wake for a horizontal offset can be neglected. Figure 9 shows the convolutions obtained with the wake functions and flattop bunches of charge equal to $1 \mathrm{nC}$ and different lengths. 


\section{CONCLUSION}

In this paper we have analytically derived expressions for the high-frequency longitudinal and transverse resistive-wall coupling impedance of an elliptical crosssection vacuum chamber. Then, the corresponding longitudinal and transversal wake functions have been obtained by calculating numerically the inverse Fourier transformations of the impedances. Once the longitudinal wake function was known, the energy changes per unit length induced within particle bunches was estimated by means of a convolution between the wake function and several flattop charge distributions. The results show that the energy variation induced within the bunch could assume an unacceptably large value when shorter electron bunches are used. Using the aluminum as material and an elliptical shape with aspect ratio $a / b>3$ for the vacuum chamber, the amplitude and the number of oscillations can be reduced.

As for the longitudinal case, the transverse kick for unit length and for transverse offset received by the particles in the vertical plane within the bunch is obtained through the convolution between the transverse wake function and the flattop charge distributions. For the particle bunches considered, the results have shown that the kick angle received from the head and tail of the bunch itself maintains an acceptable value even for shorter lengths of the charge distributions. It is worthwhile mentioning that in a small region around the axis the longitudinal wake function remains approximately constant while the transverse wake function depends linearly on the displacements of the leading and trailing charges.

\section{ACKNOWLEDGMENTS}

We thank C. Bontoiu for discussions on the FERMI@Elettra undulator vacuum chamber and for carefully reading the manuscript.

\section{APPENDIX A: ELLIPTICAL CYLINDRICAL COORDINATES}

With reference to Fig. 2, the Cartesian coordinates $(x, y, s)$ and the elliptical coordinates $(u, v, s)$ are related as follows:

$$
\begin{aligned}
& x=l \cosh u \cos v, \\
& y=l \sinh u \sin v,
\end{aligned}
$$

where $l=\sqrt{a^{2}-b^{2}}$ is the half focal length.

The metric coefficients for the elliptic cylindrical coordinates are

$$
h=h_{u}=h_{v}=l \sqrt{\cosh ^{2} u-\cos ^{2} v} .
$$

The inverse of (A1) and (A2) is

$$
\begin{aligned}
& u=\mathfrak{R}\left(\operatorname{acosh} \frac{x+i y}{l}\right), \\
& v=\mathfrak{s}\left(\operatorname{acosh} \frac{x+i y}{l}\right) .
\end{aligned}
$$

The unit vectors $\hat{u}$ and $\hat{v}$ are expressed as

$$
\begin{gathered}
\hat{u}=\frac{\hat{x} \sinh u \cos v+\hat{y} \cosh u \sin v}{\sqrt{\cosh ^{2} u-\cos ^{2} v}}, \\
\hat{v}=\frac{-\hat{x} \cosh u \sin v+\hat{y} \sinh u \cos v}{\sqrt{\cosh ^{2} u-\cos ^{2} v}} .
\end{gathered}
$$

The segment $u=0$ connects the foci, and the couples $(0, v)$ and $(0,-v)$ represent the same point on the cross section. Note that the unit vectors $\hat{u}$ and $\hat{v}$ are discontinuous on the segment $u=0$. Precisely,

$$
\begin{aligned}
& \lim _{v \rightarrow x} \hat{u}(0, v)=-\lim _{v \rightarrow-x} \hat{u}(0, v), \\
& \lim _{v \rightarrow x} \hat{v}(0, v)=-\lim _{v \rightarrow-x} \hat{v}(0, v) .
\end{aligned}
$$

\section{APPENDIX B: DERIVATION OF THE LONGITUDINAL FIELDS IN THE VACUUM REGION}

Deriving Eq. (4a) on $v(u)$ and Eq. (4b) on $u(v)$ and then summing (subtracting), we obtain the Laplace equation:

$$
\frac{\partial^{2} \tilde{E}_{z}}{\partial u^{2}}+\frac{\partial^{2} \tilde{E}_{z}}{\partial v^{2}}=0, \quad \frac{\partial^{2} \tilde{B}_{z}}{\partial u^{2}}+\frac{\partial^{2} \tilde{B}_{z}}{\partial v^{2}}=0 .
$$

Now let $F_{z}$ be the generic of the two unknowns $\tilde{E}_{z}$ and $\tilde{B}_{z}$, thus

$$
\frac{\partial^{2} F_{z}}{\partial u^{2}}+\frac{\partial^{2} F_{z}}{\partial v^{2}}=0
$$

$F_{z}$ must be $2 \pi$-periodic in $v$, it can be expanded as Fourier series on $v$ as

$$
F_{z}=F_{z 0}(u)+\sum_{n=1}^{+\infty}\left(F_{z n}^{c}(u) \cos n v+F_{z n}^{s}(u) \sin n v\right) .
$$

Substituting (B3) into (B2) yields the equation

$$
\begin{array}{r}
F_{z 0}^{\prime \prime}(u)+\sum_{n=1}^{+\infty}\left[F_{z n}^{c^{\prime \prime}}(u)-n^{2} F_{z n}^{c}(u)\right] \cos n v \\
+\sum_{n=1}^{+\infty}\left[F_{z n}^{s^{\prime \prime}}(u)-n^{2} F_{z n}^{s}(u)\right] \sin n v=0,
\end{array}
$$

where $F^{\prime \prime}(u)$ denotes the second derivative on $u$.

The latter equation is equivalent to the following system of infinitely many equations:

$$
F z_{0}^{\prime \prime}(u)=0
$$




$$
\begin{gathered}
F_{z n}^{c^{\prime \prime}}(u)-n^{2} F_{z n}^{c}(u)=0 \quad n=1,2 \ldots, \\
F_{z n}^{s^{\prime \prime}}(u)-n^{2} F_{z n}^{s}(u)=0 \quad n=1,2 \ldots
\end{gathered}
$$

whose solutions are

$$
\begin{gathered}
F z_{0}(u)=A_{0}+A_{0 u} u, \\
F_{z n}^{c}(u)=A_{n} \cosh n u+B_{n} \sinh n u, \\
F_{z n}^{s}(u)=C_{n} \cosh n u+D_{n} \sinh n u .
\end{gathered}
$$

Substituting into (B3) the longitudinal components can be written as

$$
\begin{aligned}
\tilde{E}_{z}= & A_{0}+A_{0 u} u+\sum_{n=1}^{+\infty} A_{n} \cosh n u \cos n v \\
& +B_{n} \sinh n u \sin n v+E_{n} \cosh n u \sin n v \\
& +F_{n} \sinh n u \cos n v \\
\tilde{B}_{z}= & B_{0}+B_{0 u} u+\sum_{n=1}^{+\infty} C_{n} \cosh n u \cos n v \\
& +D_{n} \sinh n u \sin n v+G_{n} \cosh n u \sin n v \\
& +H_{n} \sinh n u \cos n v .
\end{aligned}
$$

As the two couples $(0,-v),(0, v)$ specify the same point of the segment $u=0$ connecting the foci of the elliptical cross section, we must impose the condition

$$
F_{z}(0, v)=F_{z}(0,-v) .
$$

This implies $C_{n}=0$ and $H_{n}=0$. Taking this into account and substituting (B11a) and (B11b) into (4a) and (4b) yields $A_{n}=-c D_{n}, B_{n}=c C_{n}, \quad E_{n}=c H_{n}=0, \quad F_{n}=$ $-c G_{n}=0, A_{0 u}=0$, and $B_{0 u}=0$. Thus, (B11a) and $(\mathrm{B} 11 \mathrm{~b})$ reduce to $(5 \mathrm{a})$ and $(5 \mathrm{~b})$, respectively.

\section{APPENDIX C: DERIVATION OF THE TRANSVERSE FIELDS IN THE VACUUM REGION}

Differentiating and combining Eqs. (4c) and (4d) yields the equations

$$
\begin{aligned}
\frac{\partial^{2} h \tilde{E}_{u}}{\partial u^{2}}+\frac{\partial^{2} h \tilde{E}_{u}}{\partial v^{2}}= & \frac{q}{\epsilon_{0}} \delta^{\prime}\left(u-u_{1}\right) \delta\left(v-v_{1}\right) \\
& -i k \frac{\partial h^{2} \tilde{E}_{z}}{\partial u}+\frac{i}{k} \frac{\partial}{\partial u} \frac{\partial^{2} \tilde{E}_{z}}{\partial v^{2}} \\
& -i k \frac{\partial h^{2} c \tilde{B}_{z}}{\partial v}+\frac{i}{k} \frac{\partial}{\partial v} \frac{\partial^{2} c \tilde{B}_{z}}{\partial v^{2}}, \\
\frac{\partial^{2} h c \tilde{B}_{u}}{\partial u^{2}}+\frac{\partial^{2} h c \tilde{B}_{u}}{\partial v^{2}}= & -\frac{q}{\epsilon_{0}} \delta\left(u-u_{1}\right) \delta^{\prime}\left(v-v_{1}\right) \\
& +i k \frac{\partial h^{2} \tilde{E}_{z}}{\partial v}-\frac{i}{k} \frac{\partial}{\partial v} \frac{\partial^{2} \tilde{E}_{z}}{\partial v^{2}} \\
& -i k \frac{\partial h^{2} c \tilde{B}_{z}}{\partial u}+\frac{i}{k} \frac{\partial}{\partial u} \frac{\partial^{2} c \tilde{B}_{z}}{\partial v^{2}} .
\end{aligned}
$$

Deriving Eqs. (4a) and (4b) yields

$$
\begin{gathered}
\frac{\partial^{2}}{\partial v^{2}} \frac{\partial \tilde{E}_{z}}{\partial u}+\frac{\partial^{2}}{\partial v^{2}} \frac{\partial c \tilde{B}_{z}}{\partial v}=0, \\
\frac{\partial^{2}}{\partial v^{2}} \frac{\partial c \tilde{B}_{z}}{\partial u}-\frac{\partial^{2}}{\partial v^{2}} \frac{\partial \tilde{E}_{z}}{\partial v}=0 .
\end{gathered}
$$

Furthermore, using Eqs. (4a) and (4b) the following equalities can be verified:

$$
\begin{aligned}
& \frac{\partial h^{2} \tilde{E}_{z}}{\partial u}+\frac{\partial h^{2} c \tilde{B}_{z}}{\partial v}=c \tilde{B}_{z} \frac{\partial h^{2}}{\partial v}+\tilde{E}_{z} \frac{\partial h^{2}}{\partial u}, \\
& \frac{\partial h^{2} \tilde{E}_{z}}{\partial v}-\frac{\partial h^{2} c \tilde{B}_{z}}{\partial u}=-c \tilde{B}_{z} \frac{\partial h^{2}}{\partial u}+\tilde{E}_{z} \frac{\partial h^{2}}{\partial v} .
\end{aligned}
$$

Finally, substituting (C3a) into (C1a) and (C3b) into (C1b), and using (C2a), (C2b), and (A3) we obtain

$$
\begin{aligned}
\frac{\partial^{2} h \tilde{E}_{u}}{\partial u^{2}}+\frac{\partial^{2} h \tilde{E}_{u}}{\partial v^{2}}= & \frac{q}{\epsilon_{0}} \delta^{\prime}\left(u-u_{1}\right) \delta\left(v-v_{1}\right) \\
& -i k l^{2} c \tilde{B}_{z} \sin 2 v-i k l^{2} \tilde{E}_{z} \sinh 2 u, \\
\frac{\partial^{2} h c \tilde{B}_{u}}{\partial u^{2}}+\frac{\partial^{2} h c \tilde{B}_{u}}{\partial v^{2}}= & -\frac{q}{\epsilon_{0}} \delta\left(u-u_{1}\right) \delta^{\prime}\left(v-v_{1}\right) \\
& -i k l^{2} c \tilde{B}_{z} \sinh 2 u+i k l^{2} \tilde{E}_{z} \sin 2 v .
\end{aligned}
$$

The homogeneous equations associated with (C4a) and (C4b) are Laplace equations. Their solutions are denoted by $K_{\tilde{E} u}$ and $K_{\tilde{B} u}$, respectively, and have the form in (B11) with $E_{z}$ and $B_{z}$ replaced by $h \tilde{E}_{u}$ and $h \tilde{B}_{u}$, respectively.

In order to find particular solutions of Eqs. (C4a) and (C4b) in the presence of only the longitudinal fields in the right-hand sides, we substitute $\tilde{E}_{z}$ and $c \tilde{B}_{z}$ [given by (5a) and $(5 b)$ respectively] into $(\mathrm{C} 4 \mathrm{a})$ and $(\mathrm{C} 4 \mathrm{~b})$. Then the righthand sides of $(\mathrm{C} 4 \mathrm{a})$ and $(\mathrm{C} 4 \mathrm{~b})$ become linear combinations

TABLE II. The terms related to the longitudinal components in the right-hand sides of Eqs. (C4) are proportional to the functions in the Forcing term column. The corresponding particular solutions are reported in the other column.

\begin{tabular}{lc}
\hline \hline Forcing term & Particular solution \\
\hline $\sinh 2 u$ & $\frac{\sinh 2 u}{4}$ \\
$\sinh 2 u \cosh u \cos v$ & {$\left[\frac{\sinh 3 u}{16}+\frac{1}{4} u \cosh u\right] \cos v$} \\
$\sinh 2 u \cosh n u \cos n v$ & {$\left[\frac{\sinh (n+2) u}{8 n+8}+\frac{\sinh (n-2) u}{8 n-8}\right] \cos n v$} \\
$\sinh 2 u \sinh u \sin v$ & {$\left[\frac{\cosh 3 u}{16}-\frac{1}{4} u \sinh u\right] \sin v$} \\
$\sinh 2 u \sinh n u \sin n v$ & {$\left[\frac{\cosh (n+2) u}{8 n+8}+\frac{\cosh (n-2) u}{8 n-8}\right] \sin n v$} \\
$\sin 2 v$ & $-\frac{\sin 2 v}{4}$ \\
$\sin 2 v \cosh u \cos v$ & $-\frac{\sin 3 v}{16} \cosh u+u \frac{\sin v}{4} \sinh u$ \\
$\sin 2 v \cosh n u \cos n v$ & {$\left[-\frac{\sin (n+2) v}{8 n+8}-\frac{\sin (n-2) v}{8 n-8}\right] \cosh n u$} \\
$\sin 2 v \sinh u \sin v$ & $\frac{\cos 3 v}{16} \sinh u+u \frac{1}{4} \cos v \cosh u$ \\
$\sin 2 v \sinh n u \sin n v$ & {$\left[\frac{\cos (n+2) v}{8 n+8}+\frac{\cos (n-2) v}{8 n-8}\right] \sinh n u$} \\
\hline \hline
\end{tabular}


of the forcing terms in Table II, so that the problem reduces to that of finding particular solutions of the equations

$$
\frac{\partial^{2} F(u, v)}{\partial u^{2}}+\frac{\partial^{2} F(u, v)}{\partial v^{2}}=g(u, v)
$$

where $g(u, v)$ is the generic forcing term. For each a particular solution is reported in Table II. Particular solutions related to the longitudinal fields are given by $(7 \mathrm{c})-$ (7f).

Substituting the solutions $K_{\tilde{E} u}, K_{\tilde{B} u}$ and (7c)-(7f) into the first order system of Eqs. (4c) and (4d), yields the conditions $A_{0 u}=0, B_{0 u}=0, A_{n}=c D_{n}, B_{n}=-c C_{n}$, $E_{n}=-c H_{n}-\frac{n i}{k} B_{n}, \quad$ and $\quad F_{n}=c G_{n}-\frac{n i}{k} A_{n}$. Furthermore, the following conditions of continuity of the vectors $h \tilde{E}_{u} \hat{u}$ and $h c \tilde{B}_{u} \hat{u}$ must be imposed on the segment $u=0$ :

$$
\begin{aligned}
& h \tilde{B}_{u}(0, v)=-h \tilde{B}_{u}(0,-v), \\
& h \tilde{E}_{u}(0, v)=-h \tilde{E}_{u}(0,-v) .
\end{aligned}
$$

The latter conditions lead to impose: $A_{0}=0, B_{0}=0$, $A_{n}=0$, and $C_{n}=0$. In conclusion, the solutions $K_{\tilde{E} u}$ and $K_{\tilde{B} u}$ of the homogeneous Eqs. $(\mathrm{C} 4 \mathrm{a})$ and $(\mathrm{C} 4 \mathrm{~b})$ are given by (7a) and (7b), respectively. The particular solutions of $(\mathrm{C} 4 \mathrm{a})$ and $(\mathrm{C} 4 \mathrm{~b})$, related only to the charge $q$, are denoted with $S_{\tilde{E} u}^{q}$ and $S_{\tilde{B} u}^{q}$, respectively, and are obtained by starting from the following Fourier series expansion of the $u$-components of the fields and of the source terms, as follows:

$$
\begin{aligned}
& S_{\tilde{E} u}^{q}=E_{0}(u)+\sum_{n=1}^{+\infty} E_{n}^{c}(u) \cos n v+\sum_{n=1}^{+\infty} E_{n}^{s}(u) \sin n v, \\
& S_{\tilde{B} u}^{q}=B_{0}(u)+\sum_{n=1}^{+\infty} B_{n}^{c}(u) \cos n v+\sum_{n=1}^{+\infty} B_{n}^{s}(u) \sin n v,
\end{aligned}
$$

Substituting (C8a) and (C9a) into (C4a), (C8b) and (C9b) into $(\mathrm{C} 4 \mathrm{~b})$, and assuming the presence, in the right-hand sides, of only the terms related to the charge $q$, yields the following systems of equations:

$$
\begin{aligned}
& \left\{\begin{array}{l}
E_{0}^{\prime \prime}(u)=\frac{1}{2 \pi} \frac{q}{\epsilon_{0}} \delta^{\prime}\left(u-u_{1}\right) \\
E_{n}^{c^{\prime \prime}}(u)-n^{2} E_{n}^{c}(u)=\frac{q}{\epsilon_{0}} \delta^{\prime}\left(u-u_{1}\right) \frac{1}{\pi} \cos n v_{1} \\
E_{n}^{s^{\prime \prime}}(u)-n^{2} E_{n}^{s}(u)=\frac{q}{\epsilon_{0}} \delta^{\prime}\left(u-u_{1}\right) \frac{1}{\pi} \sin n v_{1},
\end{array}\right. \\
& \left\{\begin{array}{l}
B_{0}^{\prime \prime}(u)=0 \\
B_{n}^{c^{\prime \prime}}(u)-n^{2} B_{n}^{c}(u)=-\frac{n q}{\epsilon_{0}} \delta\left(u-u_{1}\right) \frac{1}{\pi} \sin n v_{1} \\
B_{n}^{s^{\prime \prime}}(u)-n^{2} B_{n}^{s}(u)=\frac{n q}{\epsilon_{0}} \delta\left(u-u_{1}\right) \frac{1}{\pi} \cos n v_{1},
\end{array}\right.
\end{aligned}
$$

for all positive integers $n$. A possible solution of system (C10) is given by

$$
\begin{aligned}
E_{0} & = \begin{cases}0 & u<u_{1} \\
\frac{q}{2 \pi \epsilon_{0}} & u>u_{1},\end{cases} \\
E_{n}^{c}(u) & =\frac{q}{\pi \epsilon_{0}} \cos n v_{1} \begin{cases}\sinh n u_{1} \sinh n u & u<u_{1} \\
\cosh n u_{1} \cosh n u & u>u_{1},\end{cases} \\
E_{n}^{s}(u) & =-\frac{q}{\pi \epsilon_{0}} \sin n v_{1} \begin{cases}\cosh n u_{1} \cosh n u & u<u_{1} \\
\sinh n u_{1} \sinh n u & u>u_{1} .\end{cases}
\end{aligned}
$$

A possible solution of system $(\mathrm{C} 11)$ is given by

$$
\begin{aligned}
B_{0} & =0, \\
B_{n}^{c}(u) & =\frac{q}{\pi \epsilon_{0}} \sin n v_{1} \begin{cases}\cosh n u_{1} \sinh n u & u<u_{1} \\
\sinh n u_{1} \cosh n u & u>u_{1},\end{cases} \\
B_{n}^{s}(u) & =\frac{q}{\pi \epsilon_{0}} \cos n v_{1} \begin{cases}\sinh n u_{1} \cosh n u & u<u_{1} \\
\cosh n u_{1} \sinh n u & u>u_{1} .\end{cases}
\end{aligned}
$$

Substituting (C12) and (C13) into (C8a) and (C8b) yields (7g) and (7h).

$h \tilde{E}_{v}$ and $h c \tilde{B}_{v}$ are straightforwardly obtained using the Eqs. (4e) and (4f).

\section{APPENDIX D: IMPOSING THE BOUNDARY CONDITIONS}

To evaluate the constants in the fields expressions, the continuity of the tangential components $\tilde{E}_{z}, \tilde{E}_{v}, \tilde{B}_{z}, \tilde{B}_{v}$ on the surface $u=u_{0}$ must be imposed.

Observing the Fourier expansion of the fields [Eqs. (7) and (9a)], it can be noticed that there is no relation between even and odd constants, because the $n$th component of the expansion depends on the $(n-2)$ th and on the $(n+2)$ th components in the vacuum, while Eq. (14) shows that angular Mathieu functions are sums of either $\pi$-periodic 
functions (even MCNs) or $2 \pi$-periodic functions (odd MCNs). Considering for $\tilde{E}_{z}$ and $\operatorname{ch} \tilde{B}_{v}$ only the terms proportional to cosines and for $\tilde{B}_{z}$ and $h \tilde{E}_{v}$ only the terms proportional to sines, only the subset of constants $A_{n}, F_{n}$, $C_{a_{n}}^{E}$, and $D_{b_{n}}^{B}$ is involved, while considering for $\tilde{E}_{z}$ and $\operatorname{ch} \tilde{B}_{v}$ only the terms proportional to sines and for $\tilde{B}_{z}$ and $h \tilde{E}_{v}$ only the terms proportional to cosines, only the subset of constants $B_{n}, E_{n}, C_{a_{n}}^{B}$, and $D_{b_{n}}^{E}$ is involved. This shows that the whole system can be divided into four independent parts.

Assuming the metal as a good conductor (like copper or aluminum) allows us to use the following asymptotic approximation for the Mathieu radial functions (which has been validated numerically):

$$
U(u) \approx e^{i\left(w_{2}-w_{1}\right)}=e^{i 2 \sqrt{Q} \sinh u},
$$

where $w_{1}=\sqrt{Q} e^{-u}$ and $w_{2}=\sqrt{Q} e^{u} . Q$ is complex, with large modulus and its square root has positive imaginary part. From Eq. (D1) the derivative of $U(u)$ on $u=u_{0}$ is given by

$$
\frac{\partial}{\partial u} U^{\prime}\left(u_{0}\right)=2 i \sqrt{Q} \cosh u_{0} U\left(u_{0}\right)=R\left(u_{0}\right) U\left(u_{0}\right) .
$$

In the sequel the subsystems will be solved, denoting with $\left(E^{<}, B^{<}\right)$the fields in the vacuum region, and with $\left(E^{>}, B^{>}\right)$the fields in the metal.

Considering the components $\cos m v$ of $\tilde{E}_{z}$ and imposing the continuity condition

$$
\left.\tilde{E}_{z}^{<}\right|_{u_{0}}=\left.\tilde{E}_{z}^{>}\right|_{u_{0}}
$$

we obtain

$$
\sum_{m=0}^{+\infty} A_{m} \cosh m u_{0} \cos m v=\sum_{n=0}^{+\infty} C_{a_{n}}^{E} U_{a_{n}}\left(u_{0}\right) \sum_{m=0}^{+\infty} A_{m}^{a_{n}} \cos m v
$$

Thus for each $m$, we can write

$$
\sum_{n=0}^{+\infty} C_{a_{n}}^{E} U_{a_{n}}\left(u_{0}\right) A_{m}^{a_{n}}=A_{m} \cosh m u_{0} .
$$

Considering the components $\sin m v$ of $c \tilde{B}_{z}$ and imposing the continuity condition

$$
\left.c \tilde{B}_{z}^{<}\right|_{u_{0}}=\left.c \tilde{B}_{z}^{>}\right|_{u_{0}}
$$

we obtain

$$
\begin{aligned}
-\sum_{m=1}^{+\infty} A_{m} \sinh m u_{0} \sin m v= & \sum_{b_{n}} D_{b_{n}}^{B} U_{b_{n}}\left(u_{0}\right) \\
& \times \sum_{m=1}^{+\infty} A_{m}^{b_{n}} \sin m v .
\end{aligned}
$$

Thus for each $m$ we can write

$$
\sum_{b_{n}} D_{b_{n}}^{B} U_{b_{n}}\left(u_{0}\right) B_{m}^{b_{n}}=-A_{m} \sinh m u_{0}
$$

We consider the $\sin m v$ components of $h \tilde{E}_{v}$ and the $\cos m v$ components of $\operatorname{ch} \tilde{B}_{v}$ on $u=u_{0}$; further, using Eqs. (17), (D2), (D4), and (D6) we write the $\cos m v$ of $h \tilde{E}_{v}^{>}$and the $\sin m v$ of $h c \tilde{B}_{v}^{>}$, in terms of the constants $A_{n}$, yielding

$$
\begin{aligned}
h \tilde{E}_{v}^{<}= & -\sum_{m=1}^{+\infty} F_{m} \cosh m u_{0} \sin m v+S_{E v}^{s} \\
& -\sum_{m=1}^{+\infty} \frac{q}{\pi \epsilon_{0}} \cos m v_{1} \cosh m u_{1} \sinh m u_{0} \sin m v, \\
h c \tilde{B}_{v}^{<}= & \sum_{m=1}^{+\infty} F_{m} \sinh m u_{0} \cos m v+S_{B v}^{c}+\frac{q}{2 \pi \epsilon_{0}} \\
& +\sum_{m=1}^{+\infty} \frac{q}{\pi \epsilon_{0}} \cos m v_{1} \cosh m u_{1} \cosh m u_{0} \cos m v, \\
h \tilde{E}_{v}^{>}= & -\sum_{m=1}^{+\infty} m \frac{i k}{\lambda^{2}} A_{m} \cosh m u_{0} \sin m v \\
& +\sum_{m=1}^{+\infty} \frac{i k}{\lambda^{2}} R\left(u_{0}\right) A_{m} \sinh m u_{0} \sin m v, \\
h c \tilde{B}_{v}^{>}= & \sum_{m=0}^{+\infty}\left[\frac{i k}{\lambda^{2}}+\frac{i}{k}\right] R\left(u_{0}\right) A_{m} \cosh m u_{0} \cos m v \\
& -\sum_{m=1}^{+\infty} m \frac{i k}{\lambda^{2}} A_{m} \sinh m u_{0} \cos m v .
\end{aligned}
$$

Finally, imposing the continuity of $h c \tilde{B}_{v}$ and $h \tilde{E}_{v}$ on $u=$ $u_{0}$ the tridiagonal linear systems (22) and (23) are obtained. Using the same arguments, the tridiagonal linear systems (24) and (25) are obtained.

[1] R.L. Gluckstern, J.V. Zeijts, and B. Zotter, Report No. CERN SL/AP 92-25, 1992.

[2] L. Palumbo and V. G. Vaccaro, Nuovo Cimento A 89, 3 (1985).

[3] A. Piwinski, Report No. DESY-94-068, 1994.

[4] A. W. Chao, Physics of Collective Beam Instabilities in High Energy Accelerators (John Wiley \& Sons, Inc., New York, 1993), pp. 38-67.

[5] H. Henke and O. Napoly, Report No. CERN/LEP-RF 8971, 1989.

[6] N.W. Ashcroft and N.D. Mermin, Solid State Physics (Harcourt Inc., New York, 1976), Chap. 1.

[7] K. Bane and M. Sands, in Proceedings of the Micro Bunches Workshop, 1995 (Report No. SLAC-PUB-957074).

[8] K. Bane and G. Stupakov, in Proceedings of 2005 Particle Accelerator Conference, Knoxville, 2005, pp. 3390-3392.

[9] K. Yokoya, Part. Accel. 41, 221 (1993).

[10] P. M. Morse and H. Feshbach, Methods of Theoretical Physics (McGraw-Hill, New York, 1953), pp. 556-568. 
[11] F. A. Alhargan, SIAM Rev. 38, 239 (1996).

[12] B. Rembold, Z. Angew Math. Mech. 53, 783 (1973).

[13] M. Abramowitz and I. A. Stegun, Handbook of Mathematica Functions (Dover, New York, 1964), pp. $722-743$.
[14] FERMI@Elettra Conceptual Design Report, Sincrotrone Trieste, 2007.

[15] R. Klatt and T. Weiland, in Proceedings of Linear Accelerator Conference, SLAC, 1986, pp. 282-285. 